
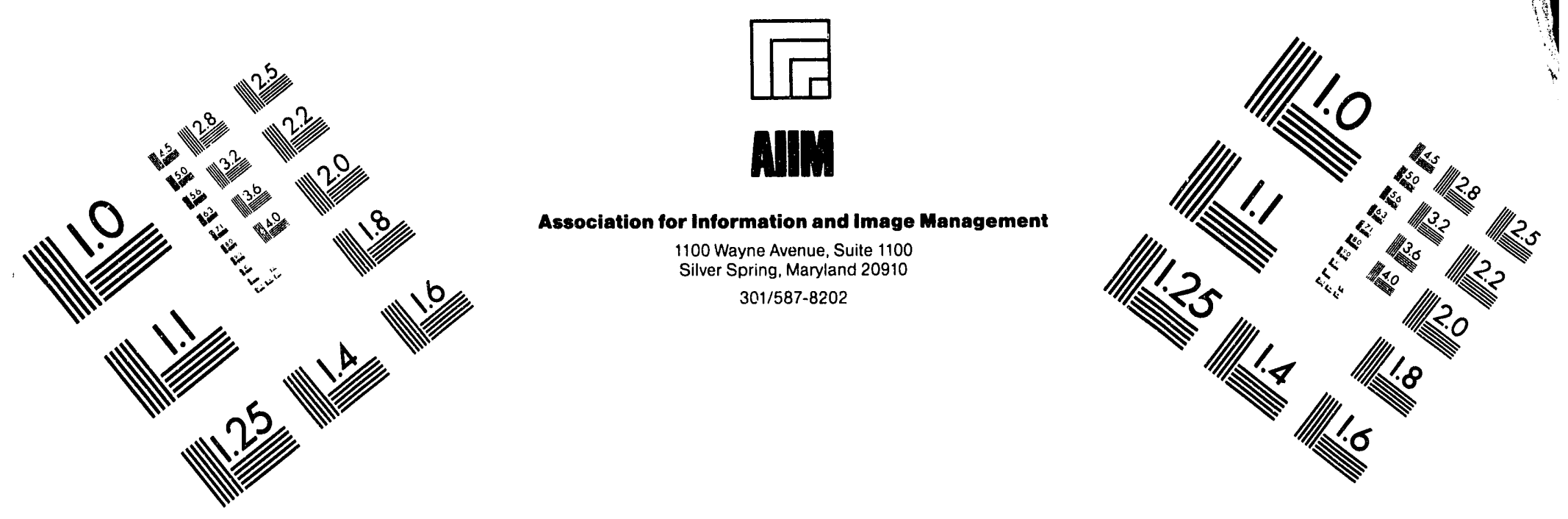

\title{
Centimeter
}

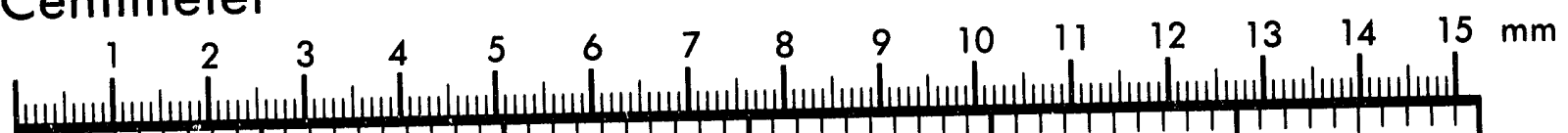

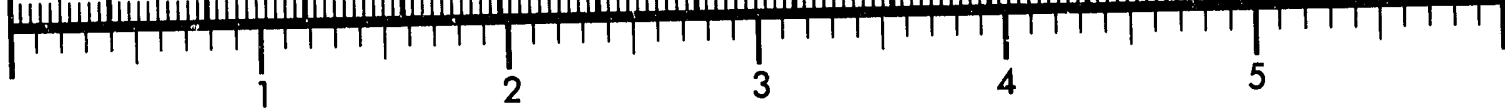
Inches
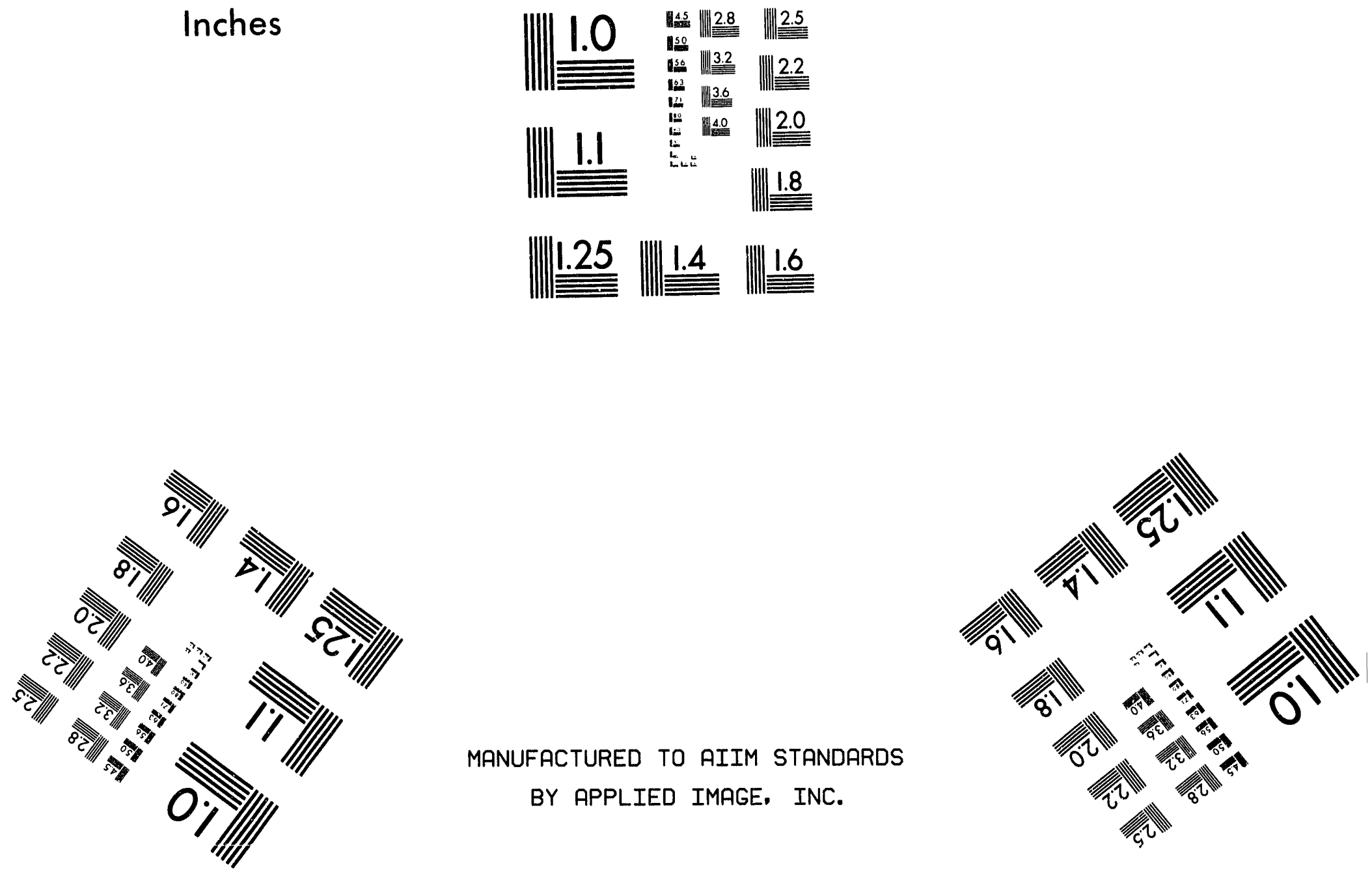

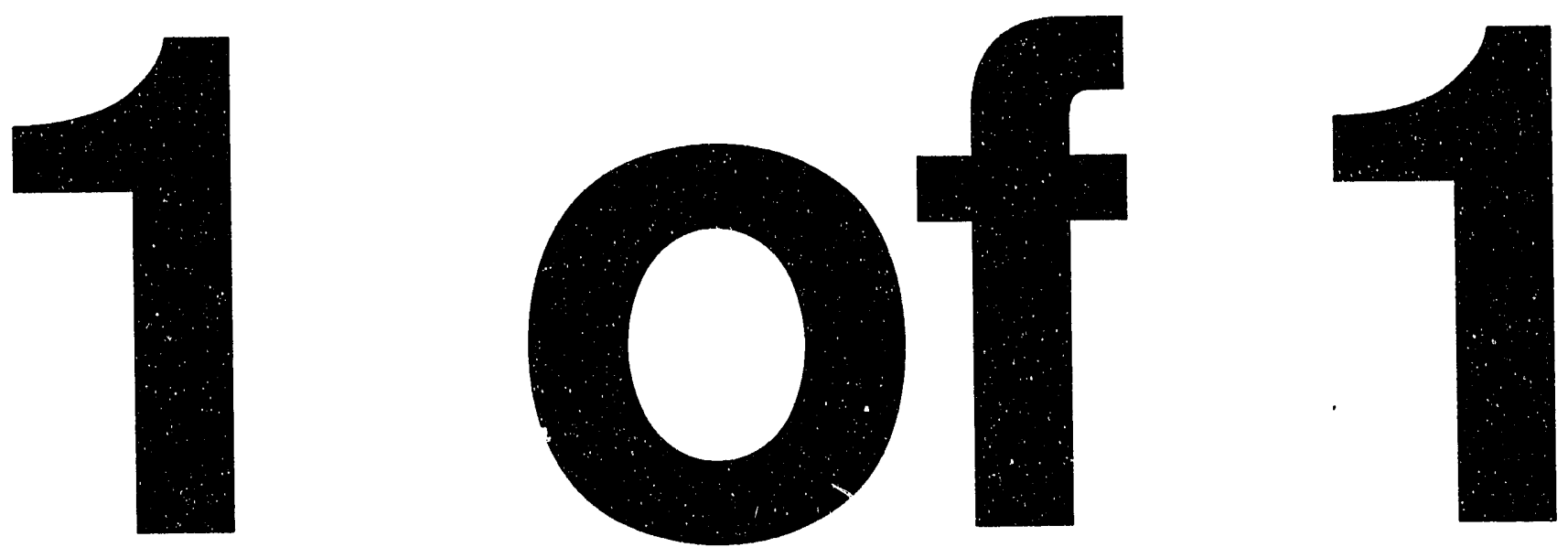


\title{
1 tef 1993
}

\section{Femtosecond Growth Dynamics of an Underdense Ionization Front Measured by Spectral Blueshifting}

\author{
Wm.M. Wood, C.W. Siders and M.C. Downer \\ Physics I lepartment. The University of Texas at \\ Austin Austin $1 \times 78712$
}

\begin{abstract}
We present a comprehensive report of time-resolved spectral blue shifts of 100femtosecond laser pulses caused by ionization of atmospheric density $\mathrm{N}_{2}$ and noble gases subjected to high $\left(10^{14} \mathrm{~W} / \mathrm{cm}^{2}-10^{16} \mathrm{~W} / \mathrm{cm}^{2}\right)$ light intensities. Included are data for two experiments: 1) self-shifting of the ionizing laser pulses for varying peak intensities, pressures (1-5 atm.), and gas species; and 2) time-resolved blueshifts of a weak copropagating probe pulse for the same range of ionization conditions. The self-shift data reveal a universal, reproducible pattern in the shape of the blueshifted spectra: as laser intensity, gas pressure, or atomic number increase, the self-blueshifted spectra develop from a near replica of the incident pulse spectrum into a complex structure consisting of two spectral peaks. The time-resolved data reveal different temporal dependence for each of these two features. We present a quantitative model for a simplified cylindrical focal geometry which explains the presence of the two spectral features in terms of two distinct ionization mechanisms: collisionless tunneling ionization, which dominates early in the ionizing pulse profile, and electron impact ionization, which dominates during the intense maximum of the ionizing pulse. Transient resonant enhancements may also contribute to ionization near the peak of the pulse.

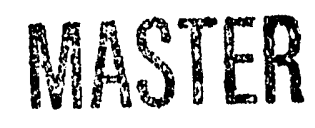

DISTRIBUTION OF THIS DOCUMENT IS UNLIMITED

Page 1 OS-92ER40739 
Femtosecond Growth Dynamics..., Wood, Siders, Downer

\title{
Femtosecond Growth Dynamics of an Underdense Ionization Front Measured by Spectral Blueshifting
}

\author{
Wm.M. Wood, C.W. Siders and M.C. Downer \\ Physics I Department. The University of Texas at
}

Austin Austin $7 X 78712$

\section{Introduction}

Interest in highly ionized plasmas approaching atmospheric density, as a potential future source of coherent $x$-rays ${ }^{1-3}$, and as a potential medium for charged particle acceleration 4.5 , has been renewed by the recent capability of creating such plasmas on femtosecond time scales ${ }^{6}$. Ionization by intense, femtosecond laser pulses holds promise for precise control of initial plasma conditions (temperature, ionization state, density) 1.7 which are critical to these applications. Theories treating the ionization rates of atoms under extremely high field conditions 8,9 can now be studied and compared directly with experiments involving laser pulses with electric field amplitudes that surpass the fields binding the electrons to atoms. Most recent experiments in this regime have been based on measurements of intensity dependent ion yields ${ }^{10}$ or photoelectron energy spectra ${ }^{11}$. Typically, such experiments require low pressure $\left(-10^{-6}-10^{-4}\right.$ torr) noble gases, in order to allow collisionless ion or electron collection and to avoid space charge effects. At these pressures, the microscopic behaviour of single atoms interacting with the laser field is revealed. On the other hand, at gas pressures in the atmospheric range inter-atomic effects, such as ionization via electron impact, and collective effects begin to play a significant role. Here, the vacuum diagnostic techniques of ion-yield and photoelectron spectroscopy can no longer be employed as tools to monitor the atomic ionization dynamics. 
At these higher gas densities, the alternative diagnostic technique of frequency upconversion, or blueshifting, caused by radiation interacting with an ionization front $12,14,15$, can be utilized to investigate the dynamics of ionization in intense laser fields. Moreover, the blueshifting technique is readily amenable to femtosecond timeresolved pump-and-probe measurements ${ }^{26}$, and can therefore provide information on the temporal dependence of ionization within the ionizing pulse. In contrast to Doppler shifting in reflection from a moving critical surface (or mirror), frequency shifts induced at a moving ionization front involve no net increase in the energy of the blueshifted pulse, and must be described differently 12,15 . In the experiments reported here, we consider a specific interaction geometry in which a focused laser pulse - either the ionizing pulse itself or a separate probe pulse of the same visible wavelength copropagates in free space with an underdense ionization front created by the ionizing pulse. In this case, the velocity of the ionization front equals the group velocity of the pulse throughout their interaction length ' 1 ', the upper limit of which is set by the Rayleigh length of the focused pulse(s). Consequently the front maintains a constant position within the temporal profile of the pulse throughout the interaction. In this case, the spectral blueshift is described most simply as a rapid decrease of the index of refraction during the pulse ${ }^{12,16}$. Specifically, the index $n$ of an underdense free electron plasma can be expressed in terms of the plasma frequency $\omega_{\mathrm{p}}$ :

$$
n\left(\omega_{0}\right)=\left(1-\frac{\omega_{p}^{2}}{\omega_{0}^{2}}\right)^{1 / 2}
$$

If the index of refraction changes during the laser pulse, the portion of the pulse experiencing the change is subjected to a frequency shift given by 12 :

$$
\Delta \omega=-\frac{\omega_{0}}{c} \int_{0}^{z} \frac{\partial n}{\partial t}(l) d l
$$


Femtosecond (;rowth Dynamics..., Wood, is: : 3 , Downer

Here, $\omega_{0}$ is the angular frequency of the incident laser, and $\mathrm{n}$ is given by expression (1) above. The spectral shift imposed on the light occurs at the moment of ionization 17,25. An alternative numerical procedure for describing the blueshift in this geometry, in which we solve Maxwell's equations for the wave propagation consistently with the electron dynamics using an electromagnetic plasma simulation code has been developed in a separate paper ${ }^{18}$. Frequency upshifts resulting from a rapidly generated overdense plasma have been discussed by Wilks et al. ${ }^{19}$. In addition to ionization fronts, traveling density waves in pre-ionized plasmas can also induce frequency shifts on copropagating light pulses, opening the possibility of novel radiation sources and diagnostic experiments on plasma wakefield oscillations 20,21 . A related frequency upshift occurring in a different interaction geometry - microwave radiation impinging upon an ionization front created in a microwave cavity by an ultraviolet pulse - has recently been predicted $^{22}$ and experimentally confirmed 23,24 . In this case, in contrast to the experiments discussed here, the microwave radiation and the ionization front propagate at substantially different velocities. Moreover, the dispersion of the microwave cavity must be taken into account. Consequently, the physics of the microwave frequency upshift in the ionization front is described differently $22,23,24$.

Femtosecond duration pulses are essential for a clean diagnosis of the ionization front in our experiments. When using laser pulses longer than several picoseconds, blueshifting of the pulse spectrum caused by plasma creation is accompanied by a red shifting due to plasma expansion out of the focal region ${ }^{12}$. The resulting spectrum appears asymmetrically broadened after the ionization. However, with laser pulses of only 100 femtosecond duration, the pulse has departed from the focal region before the plasma can expand or recombine significantly; furthermore, spectral broadening caused by non-ionizing sources of phase modulation can be rendered negligible with a sufficiently tight focal geometry. As a result, only blue shifting of the laser pulse, due to plasma creation, is observed ${ }^{6}$. In a previous Letter ${ }^{13}$ we briefly reported experiments 
which measured the self-blueshift of a femtosecond ionizing pulse in 1- to 5atmospheres noble gas samples, and femtosecond time-resolved pump-probe experiments which used the probe blueshift to measure ionization dynamics throughout the intense ionization pulse. In addition, a model based on strong-field tunneling ionization rates, supplemented by a slower collisional ionization process was briefly presented to explain the results. The main purpose of the current paper is to expand the presentation of these experimental results. Complete self-blueshift and time-resolved blueshifting data of 100femtosecond laser pulses caused by ionizing breakdown in the noble gases $\mathrm{He}, \mathrm{Ne}, \mathrm{Ar}$, $\mathrm{Kr}, \mathrm{Xe}$, and $\mathrm{N}_{2}$ at pressures ranging between 1 and 5 atmospheres are included. A second purpose is to expand upon and clarify two features of the model which were not discussed previously: 1) evaluation of the minor contribution of non-ionizing sources of phase modulation to the spectral changes under our experimental conditions; and 2) the effect of electronic excitation of target ions on the electron impact ionization crosssections used in the model. Finally, the possible contribution of transient resonant enhancements of ionization near the peak of the pump pulse is discussed qualitatively.

The paper is organized as follows: In section $\amalg$, the experimental procedure is described, followed in section III by the results of the self-blueshifting experiments over a range of pressures, peak intensities, and gases. Femtosecond, time-resolved spectral shifts are then presented. Section IV evaluates several non-ionizing sources of phase modulation. Finally, in section $\mathrm{V}$ we propose a quantitative model which relates observed blueshifted spectral features to two distinct ionization mechanisms: collisionless strong-field tunneling ionization, and electron impact ionization.

\section{Experimental Procedure}

The experiments used a $10 \mathrm{~Hz}, 90$-fs dye laser system described elsewhere 6 . Figure 1 shows the experimental set-up. The incoming 90-fs pulse with center wavelength of $620 \mathrm{~nm}$ is focused at $\mathrm{f} / 5$ through the $20 \mathrm{x}$ microscope objective (focal 
length $=1.7 \mathrm{~cm}$.) into a gas cell containing pressures ranging from 1 to 5 atmospheres of $\mathrm{He}, \mathrm{Ne}, \mathrm{Ar}, \mathrm{Kr}, \mathrm{Xe}$, or $\mathrm{N}_{2}$. For the single pulse experiments, beamsplitter at $\mathrm{A}$ is removed, and the polarizing beamsplitters at $\mathbf{B}$ and $\mathbf{C}$ are replaced by neutral density filters whose sum is constant, enabling variation of the pulse energy at the interaction while ensuring that a constant energy reaches the spectrometer. At low intensity, the minimal $e^{-1}$ focal spot diameter is measured as $7 \pm 0.5$ microns. Pulse energies are less than or equal to $0.4 \mathrm{~mJ}$. (A $90 \mathrm{fs}$. pulse of $0.4 \mathrm{~mJ}$ energy focused to $7 \pm 0.5$ micron

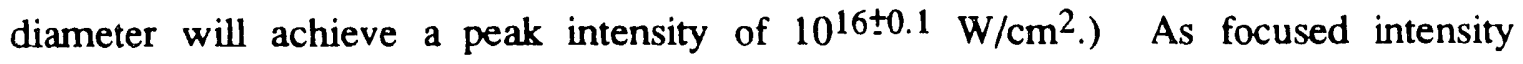
increases above the ionization threshold, an increasingly bright breakdown spark is observed, while the exiting far field beam profile increasingly expands because of defocusing in the reduced-index plasma. Nevertheless, all of the light transmitted through the focal region (typically $>95 \%$ of the incident energy) is captured by a large collection lens, and sent to the spectrometer/Optical Multichannel Analyzer via a bundle of optical fibers. Care is taken to avoid excessive intensities in the fiber bundle. The bundle itself transmits a homogeneous spatial light distribution to the spectrometer. A pulse duration measurement before and after the focus (fig. 2a) reveals that the focusing and recollimating optics broaden the initial $90 \mathrm{fs}$. pulse to $100 \mathrm{fs}$.; the presence of a spark breakdown in 5 atmospheres pressure Ar does not broaden the pulse further. Finally, the gas cell is positioned carefully with respect to the microscope objective to avoid $\chi^{(3)}$ effects in the glass walls of the cell. Absence of a spectral shift after transmission through the evacuated cell confirms that spectral shifts are due solely to the interaction of the laser with the gas.

Time-resolved investigations were carried out using $115 \mathrm{fs}$ pulses. In figure 1, the incoming laser pulse is split into a pump beam and a probing beam with approximately $4 \%$ of the beam energy. The pump beam creates the ionizing breakdown as before. The probing beam travels through a retroreflecting delay arm to vary the time of arrival of the probe with respect to the pump. The probe beam is polarized orthogonally to the pump 
beam with a zeroth-order half wave plate, then recombined with the pump beam in a polarizing beam splitting cube. The two beams co-propagate through the interaction region, with relative divergence of less than $0.1 \mathrm{mrad}$. After the interaction with the gas, the light is recollimated with the large collection lens with $n=$ gligible radiation loss, and the probe is separated from the pump beam by using a second polarizing cube at point $\mathrm{C}$. We verified that the transmission of the polarizer was negligibly affected as the exiting pump and probe beam divergences changed because of ionization-induced defocusing. The spectrum of the probe beam after the interaction is then further isolated from depolarized, scattered purmp radiation by real-time spectral background subtraction, and recorded in the optical multichannel analyzer. $T_{0}$, the temporal overlap between the pump pulse and the probing pulse, is determined to within $\sim 20$ fs. by first, purposely misaligning the recombination beamsplitter to mix the pump and probe beam polarizations, adjusting the probe delay to maximize the contrast in the resulting interference fringes, and then realigning the beamsplitter to its original position.

\section{Experimental Results}

\section{A. Self-Blueshift Data}

Fig. 2b) shows the common input pulse spectrum. Figures 3 through 6 present the measured self-blueshifts of linearly polarized, 90 -fs. pulses after ionizing each of the gases. Vertical bars indicate the center wavelength $(620 \mathrm{~nm})$ of the unshifted spectrum in each figure. Each spectrum is the result of averaging 100 laser pulses. Figures 3 and 4 show the self-blueshifted spectra as a function of increasing calculated peak intensity constant sample pressures of 1 - and 5- atmospheres, respectively. Heavy horizontal bars indicate spectra corresponding to the threshold peak intensity at which a recombination spark is first visible. In every case, this threshold coincides with both the threshold for spectral blueshifting and the onset of defocusing, confirming their common origin in 
generation of a plasma. Figure 5 shows self-shifted spectra as a function of gas pressure at constant peak intensity of $10^{16} \mathrm{~W} / \mathrm{cm}^{2}$. Figure 6 compares the shifts between the various gases measured for the highest pulse peak intensities at 1- and 5- atmospheres pressure.

Several general observations about the self-blueshifted data can be made. The threshold energy at which the recombination spark, defocusing, and the blueshift are first observed decreases as the noble gas atomic weight is increased. This can be explained by the decrease of the ionization potentials $U_{i}$ of the atoms with increasing atomic weight. For the same reason more ionization, and therefore greater blueshifting, occur as atomic weight increases. The dimer gas $\mathrm{N}_{2}$ is expected to display somewhat more complex behaviour because the ionization occurs on a time scale which dues not allow the two atoms to separate by a significant amount, and indeed does not fit exactly with these trends. The breakdown threshold pulse energy decreases in each gas as pressure is increased, because a smaller percentage of the atoms must be ionized to create an observable breakdown spark, and therefore a lower intensity is needed.

A universal, reproducible pattern in the shape of the blueshifted spectra in the heavier gases is observed. Specifically, with increasing laser intensity, gas pressure, or atomic number, the self-blueshifted spectra develop from a near replica of the incident pulse spectrum inio a complex structure consisting of two spectral peaks: a narrow peak shifted between 5 and $10 \mathrm{~nm}$ towards the blue from the original spectrum, whose position is independent of pulse energy and pressure above some threshold energy; and a broad shoulder or peak shifted further towards the blue whose position and width depend strongly on the gas pressure, gas species, and the laser pulse energy. (In $\mathrm{He}$ and $\mathrm{Ne}$, this broad blue shoulder is not observed under most of the conditions which we investigated, although the beginnings of such a shoulder can be seen in Ne at the highest pulse energy in 5 atmospheres pressure.) The breadth of the blue shoulder depends on gas species, gas pressure, and pulse peak intensity. As the breadth of the blue shoulder increases with 
increasing pulse energy, the height of the narrow, less-shifted peak decreases, indicative of a trade-off of total energy between these separate features. In both $\mathrm{Xe}$ and $\mathrm{Kr}$, the blue shoulder becomes a distinct spectral peak which is much broader, and shifted much further, than the narrow peak. Because the magnitude of the shift is proportional to the ionization rate of the gas, the presence of two distinct peaks in the shifted spectra implies the existence of two distinguishable rates of ionization during the laser pulse.

These data reveal two important clues to the underlying ionization dynamics: First, as can be seen in figure 6 , both $\mathrm{He}$ and $\mathrm{Ne}$ gases show a significant portion of the final pulse energy remaining "unshifted," i.e. remaining around $620 \mathrm{~nm}$, the center of the original pulse. At 1 atmosphere pressure, this appears as a relatively small shift of the spectral peak $(2 \mathrm{~nm})$ compared to the other gases. At 5 atmospheres pressure, this "unshifted" energy appears as a red "shoulder" on the He and Ne blueshifted spectra, with Ne displaying a smaller shoulder than He. This feature is also observed in Ar at 1-atm. In contrast, in each of the other gases, and Ar at 5-atm., this "shoulder" is completely absent, and the absence of spectral energy near $620 \mathrm{~nm}$ indicates that almost the entire pulse energy has been spectrally shifted. These observations imply that, since the spectral shifting is caused directly by the temporal modulation of the index of refraction, ionization is occurring during only part of the pulse in $\mathrm{He}$ and $\mathrm{Ne}$ gases, whereas ionization is occurring during the entire pulse for the other gases.

Several other observations bear comment. First, the appearance of a breakdown spark and spectral blueshifting is always accompanied by a defocusing of the laser beam leaving the interaction. The degree of defocusing is correlated with the amount of ionization occurring in the sample, ranging from only a slight change in the emerging beam profile in the case of $\mathrm{He}$ at 1 atmosphere pressure, to a greater than $3 \mathrm{x}$ expansion of the outgoing cone of light in the case of $5 \mathrm{~atm}$. Xe. Also in each case, the spatial profile of the emerging laser pulse is changed from a nearly gaussian profile before the experiment ${ }^{6}$ to a ring profile after the ionization. A second observation is that the use of 
circularly polarized light to ionize the gases, measured by inserting a quarter wave plate into the beam path before the initial focusing microscope objective, reveal shifted spectra which are indistinct from those measured using linearly polarized light.

\section{B. Time-Resolved Data}

Figure 7 shows the autocorrelation for the pulses used in the time-resolved experiment, which had a somewhat longer pedestal than the shortest pulse measured from the amplifier (fig. 2b.) Figures 8 and 9 show the measured time-resolved probe spectra in 1 - and 5- atmospheres pressure, respectively, using a (calculated) peak pump pulse intensity of $10^{16} \mathrm{~W} / \mathrm{cm}^{2}$. The time delay between the ionizing pulse and the probe pulse varied between $-640 \mathrm{fs}$. and $480 \mathrm{fs}$. Each spectrum is the average of 50 laser pulses. In each figure, time increases towards the top, with negative times referring to the probe pulse preceding the ionizing pulse, and positive times to the probe trailing the ionizing pulse. Time $t=0$, corresponding to exact pump-probe overlap, is demarcated further by an emboldened spectrum. In each of the series of spectra, the spectral shift first appears as a second peak towards the blue of the original spectrum, at times between -500 and $400 \mathrm{fs}$. This early appearance is caused by ionization in the energy "pedestal" of the ionizing pulse. As the probe peak approaches but still precedes the ionizing pulse peak, both the broad, blue shoulder and the narrow less-shifted peak appear (except in He and $\mathrm{Ne}$, where the shoulder never appears.) In each gas significant blueshifting occurs early in the pump pulse. When the pump and probe pulses temporally coincide, the probe spectra closely resemble the self-shifted spectra. When the probe trails the ionizing pulse, the blue "shoulder" disappears from the probe spectra, whereas the narrow, lessshifted peak remains. After $300-400 \mathrm{fs}$, the probe spectra return to the original spectrum centered at $620 \mathrm{~nm}$.

To facilitate analysis of these time-resolved data, figures 10 and 11 have been created, representing the integrated energy in each of the time-resolved probe spectra in 
small bands around $610 \mathrm{~nm}$ and $615 \mathrm{~nm}$. These plots display the different temporal evolution of each of the two parts of the spectra, in each of the gases. re temporal evolution of the narrow, less-shifted peak is revealed by the curve at $615 \mathrm{~nm}$, whereas the temporal dependence of the broad, blue shoulder is shown by the curve at $610 \mathrm{~nm}$. (The temporal behaviour at wavelengths $<610 \mathrm{~nm}$ is the same as that at $610 \mathrm{~nm}$ to within limits of instrument sensitivity) Within each graph, the spectral energy near $610 \mathrm{~nm}$ consistently peaks well before the most intense part of the ionizing pulse at $t=0$. In He and $\mathrm{Ne}$ gases at 1 atm., this peak occurs nearly 300 fs before $t=0$. In each of the other gases, the maximum of the $610 \mathrm{~nm}$ feature occurs between 200 and 100 fs before $t=0$. The spectral energy at $615 \mathrm{~nm}$, on the other hand, peaks well after the $610 \mathrm{~nm}$ feature. This means that the index modulation giving rise to the narrow, less-shifted peak occurs after that process giving rise to the broad, blue shoulder in each gas. In $\mathrm{Ar}, \mathrm{Kr}, \mathrm{Xe}$ and $\mathrm{N}_{2}$ gases at $5 \mathrm{~atm}$., and in $\mathrm{Kr}$ and $\mathrm{Xe}$ at $1 \mathrm{~atm}$., this peak of the $615 \mathrm{~nm}$ feature lies between $50 \mathrm{fs}$ and $100 \mathrm{fs}$ after $\mathrm{t}=0$. In $\mathrm{Ar}$ and $\mathrm{N}_{2}$ at 1 atm., and $\mathrm{Ne}$ and $\mathrm{He}$ at $5 \mathrm{~atm}$., this peak is right about at $\mathrm{t}=0$. In both $\mathrm{He}$ and $\mathrm{Ne}$ at $1 \mathrm{~atm}$., blueshifting maximizes before $\mathrm{t}=0$ at all wavelengths.

\section{Non-ionizing sources of phase modulation}

Before proceeding with a model of our experimental blueshifts based solely on ionization, we first discuss the possible role of non-ionizing sources of phase modulation. In all of the above data, we consistently observe a pure spectral shift of the spectrum of the tightly focused pump or probe pulse. Without exception, new spectral components arise to the blue side of the original pulse spectrum, while components to the red side are, within measurement sensitivity, entirely absent. This contrasts sharply with the wide spectral broadening, or supercontinuum generation, produced by interaction of intense short pulses with neutral noble gases in longer focal geometries when ionization was minimal or negligible 27 . The basic explanation for this contrast is that ionization induces 
a temporally monotonic reduction in refractive index from $n \approx 1$ to $n=\left(1-\left(\omega_{p}{ }^{2} / \omega_{0}{ }^{2}\right)\right)^{1 / 2}$, whereas the nonlinear index $\mathrm{n}_{2}$ of the neutral gas induces a temporally symmetric index modulation $n_{2} I(t)$, which rises, then falls, with the pulse intensity envelope $I(t)$. The observation of pure blue shifts, coincident with recombination luminescence and defocusing, thus provides prima facie evidence that ionization is the dominant source of phase modulation in our experimental geometry. Conversely, self-focusing (often evider. in "filamentation" of the transmitted pulse), which has a common origin with spectral broadening in the nonlinear index $\mathrm{n}_{2} \mathrm{I}$ of the gas, is completely absent. In addition, in proceeding through the noble gas series, we observe a smooth progression of increasing blueshifts despite $n_{2}$ values which vary by a factor of more than 200 . Finally, Corkum et. al. ${ }^{27}$ observed no supercontinuum from Ne under any conditions, whereas its blueshift fits in smoothly with the noble gas sequence under ionization conditions.

We now present theoretical estimates which confirm that phase modulation from ionization should indeed strongly dominate non-ionizing sources under our experimental conditions. Four non-ionizing sources of phase modulation could potentially play a role in the experiment: Prior to ionization, the laser experiences the nonlinear refractive index $\left(n_{2}{ }^{\text {neutral }}\right)=\left(2 \pi / n_{0}\right) \chi^{(3)}(-\omega, \omega, \omega,-\omega)$ of the neutral gas, where $n_{0}-1$ is the linear index of the neutral gas and $\chi^{(3)}$ is its third order nonlinear susceptibility. While the gas remains neutral, the total index varies as $n(t)=n_{0}+\left(n_{2}\right.$ neutral $) I(t)$, resulting in a phase modulation $\phi(t)=n(t) \omega / c$. After ionization, the neutral gas disappears, but the resuiting plasma gives rise to a new nonlinear index $\left(n_{2}\right.$ plasma $)=\left(n_{2}{ }^{\text {ion_core }}\right)+\left(n_{2}\right.$ free_electron $)+$ ( $\mathrm{n}_{2}$ collective) which is a summed contribution of three additional sources of modulation: 2) The nonlinear index ( $\left.n_{2}{ }^{\text {ion_core }}\right)$ from the hyperpolarizability of the ionic cores, 3 ) the nonlinear index $\mathrm{n}_{2}$ free_electron) arising from relativistic motion of the free electrons in the strong light field, and 4$)$ nonlinearities $\left(\mathrm{n}_{2}\right.$ collective) arising from collective ionic or electronic motion induced by ionization or ponderomotive pressure of the light pulse. Nonlinear optical effects from free electron motion can be ruled out immediately. These 
become significant only when the $\mathrm{v} \times \mathrm{B}$ force from the light magnetic field becomes comparable to the electric force ${ }^{28}$. This occurs when the electrons are driven relativistically, corresponding to pulse peak intensities above approximately $10^{18} \mathrm{~W} / \mathrm{cm}^{2}$ at visible wavelengths, much higher than our intensities. Similarly, nonlinear optical interactions of the light with collective ion acoustic modes can be neglected, since these modes require acoustic time scales to become significant ${ }^{29,30}$, much longer than the pulse duration. Moreover, collective electron oscillations induced by ionization or pondermotive pressure, can be shown to be too small in magnitude and too high in frequency under our experimental conditions to modulate the light pulse significantly 31 . Thus we concentrate on evaluating ( $\left.\mathrm{n}_{2}^{\text {neutral }}\right)$ and ( $\left.\mathrm{n}_{2}^{\text {ion_core }}\right)$.

We first compare the ionization-induced index modulation near the ionization threshold directly with the hypothetical index modulation $\left(\mathrm{n}_{2}{ }^{\text {neutral }}\right) \mathrm{I}(\mathrm{t})$ which would be induced by the same light intensity in the neutral gas. Following a closely analogous calculation by Dawes 32 for the linear susceptibilities $\chi(\omega)$ and the third harmonic coefficients $\chi^{(3)}(-3 \omega, \omega, \omega, \omega)$ of the noble gases, we calculate the approximate contribution to the nonlinear index change at the ionization threshold. We use the approximations i) that the atoms are in their electronic ground state and ii) that the laser photon eneigy $h \omega=2.0 \mathrm{eV}$ is much less than the energy of any atomic resonances, thus allowing all excited state energy denominators to be approximated by a common denominator $E_{0}=h \omega_{0}$, which in turn is very clos: $o$ the ionization potential. In close analogy to Dawes' result, these approximations lead to a straightforward formula:

$$
\chi^{(3)}(\omega,-\omega, \omega,-\omega)=\frac{N_{0} e^{4} h^{4}}{3 m^{2}} \frac{5 E_{0}^{2}-2 E_{\omega}^{2}}{E_{0}\left(E_{0}^{2}-4 E_{\omega}^{2}\right)\left(E_{0}{ }^{2}-E_{\omega}{ }^{2}\right)^{2}}
$$

where Dawes treats $\mathrm{E}_{0}$ and $\mathrm{N}_{\mathrm{o}}$ as fitting parameters, the latter representing an effective number of electrons. Since Dawes showed that the $\chi^{(1)}(\omega)$ and $\chi^{(3)}(-3 \omega, \omega, \omega, \omega)$, calculated with common fitted parameters, agreed with measured values 33,34 in the 
Femtosecond Growth Eynamics..., Wood, Siders, Downer

noble gases to within an experimental error of $\pm 50 \%$, we can expect comparable accuracy for our $\chi^{(3)}(-\omega, \omega, \omega,-\omega)$ values, for which we adopt Dawes' fitted values without change. The resulting calculated ratios of the neutral gas nonlinear index change $\left(\Delta \mathrm{n}^{\mathrm{n} .1 .}=2 \pi \mathrm{N}_{\mathrm{o}} \chi^{(3)} \mathrm{E}^{2}\right)$ to the ionization-induced index change ( $\Delta$ piasma $=2 \pi \mathrm{N}_{0}$ $\left.\mathrm{e}^{2} / \mathrm{m} \omega^{2}\right)$, assuming an intensity of $10^{16} \mathrm{~W} / \mathrm{cm}^{2}$, are shown in the first row of Table 1 . Subsequent rows show the estimated ratios for ions of different charge, in the ground state. These estimates show that, within the intensity range of our experiments, the contributions to the index changes should be dominated by plasma effects.

The ratio of ( $\left.\Delta n^{\text {ion_core }}\right)$ to ( $\left.\Delta n^{\text {plasma }}\right)$ can also be estimated from more general arguments, which are not restricted to assuming the atoms/ions are in the ground electronic state. This is done by finding an upper limit to this contribution and comparing it with the contribution from the free electrons. Since we only need an upper limit on the electron motion, we consider the most loosely bound electrons as classical oscillators. The polarization can be written:

$$
P(\omega)=N_{0} \operatorname{ex}(\omega)
$$

The position $x(\omega)$ of the electron can be expanded in terms of the harmonics of the field:

$$
x(t)=\sum_{n} x(n \omega) \cos \left(n \omega t+\phi_{n}\right)
$$

Because we are only interested in the index of refraction at the fundamental frequency, and not in harmonic generation, we need only consider the contributions at $\omega$. Requiring that the electron remains bound entails that the kinetic energy of the oscillator remain less than the negative of the electric potential throughout the electron trajectory. This results in an upper limit on the electron amplitude before it must become free:

$$
x(\omega)<10.94 \sqrt[3]{\frac{Z}{\omega^{2}}}
$$


Here, $\mathrm{Z}$ is the net charge of the ion binding the electron in question, and ' $\mathrm{x}$ ' is in meters. In eqn (4), this amplitude provides an upper limit on the total contribution to the polarization from a single, bound electron.

Because the polarization from the $\omega$-term in equation (5) includes contributions from any nonlinear terms (e.g. $\chi^{(3)}, \chi^{(5)}$,) we will over-estimate the amplitude of an electron trajectory if we suppose that the amplitude of oscillation decreases linearly with the laser electric field. It is now assumed that the electric field giving rise to the maximum amplitude of expression (6) corresponds to the ionization threshold value in the Ammosov formulation of strong-field ionization. Graphical analysis of the formula given by Ammosov ${ }^{9}$ yields the electric field amplitude of linearly polarized light required to strip an electron from the atom. Using $620 \mathrm{~nm}$ radiation, the probability of ionization in one light cycle $2 \pi / \omega$ exceeds unity when:

$$
\frac{\left|E_{\text {applied }}\right|}{\left|E_{\text {atom }}\right|}>0.1
$$

The electric field in the classical atom scales as the square of the ionization energy. This allows us to use the tabulated ionization energies to estimate $E_{\text {atom }}$ in expression (7). Adding the contributions from each electron yields the estimate on the upper limit for the bound electron polarization. We compare the ratio of this limit to the contributions from the free electrons for various stages of ionization in Table 2.

Comparison of the values found in Table 1 with those in Table 2 indicate that the actual values for bound electron contributions to the polarization will be much smaller than our upper limit. Only in the case of resonance might the limit be approached; in this case we expect such resonances to be too transient to affect the pulse significantly 35 . Also, in atoms where bound electrons might have an effect comparable to the free electrons, computer modelling has shown that pulses with peak intensity above $10^{15}$ $W / \mathrm{cm}^{2}$ ionize the atoms early enough during the pulse so that only a small portion of the 
Femtosecond Growth Dynamics..., Wood, $₫ \triangleleft .$, s, Downer

light energy would be affected. This is an especially important observation when considering pulse propagation through the plasma region. Ii can be concluded that any phase modulation caused by bound electrons during the early part of the ionizing pulse will be dominated by the ionization effects. As an aside, it would be a very interesting experiment to investigate a regime (in singly ionized $\mathrm{Xe}$, or perhaps a heavy alkaline earth such as $\mathrm{Ba}$ ) where nonlinear effects might possibly dominate free electron effects on the polarization.

\section{Model of Ionization-Induced Phase Modulation}

We now present a quantitative model of the blueshifts based solely on ionization, neglecting other sources of phase modulation. The model accounts for the major experimental observations: 1) the narrow, less shifted peak and the broad blue shoulder observed in $\mathrm{Ar}, \mathrm{Kr}$, and $\mathrm{Xe}, 2)$ the contrasting temporal behaviour of these two universal features in the shified spectra, and 3) the unshifted portions of the spectra observed in He and $\mathrm{Ne}$. The central concept of the model is that the ionization rate under these experimental conditions is governed by two separate mechanisms. The first is collisionless, radiative ionization of each atom solely through its interaction with the intense light field. A number of theories based on quantum mechanical tunneling 8,9 or Coulomb barrier suppression ${ }^{10}$ have been used to describe this process in low density media. Regardless of which theoretical model is used, however, calculations show that for the noble gases, this solely strong-field process saturates early in the temporal profile of the focused ionizing pulses, and thus can explain only the blueshift of the leading edge. To explain the persistence of ionization, and therefore blueshifting, into the peak of the pulse, a second ionization mechanism must be invoked. In the following discussion, we will consider two possible mechanisms for the "extra" ionization near the peak of the pulse: 1) impact ionization induced by electrons freed early in the pulse, which derive oscillatory impact energy from their quiver motion in the intense light field. 
Femtosecond Growth Dynamics..., Wood, Siders, Down::1

This model will be discussed in quantitative detail. 2) Resonant enhancement of ionization as ionic excited states are AC Stark shifted into resonance with a harmonic of the light field ${ }^{11}$. This model will be discussed only qualitatively. The current experimental results do not permit definitive disconfirmation of either model, but do strongly support the existence of extra non-tunneling ionization near the peak of the pulse.

It is not difficult to see why the first (collisional) mechanism can become important near the peak of the pulse. In a light field of $10^{16} \mathrm{~W} / \mathrm{cm}^{2}$, the amplitude $2 \mathrm{eE} / \mathrm{m} \omega^{2}-75 \AA$ of quiver motion exceeds the average interatomic spacing $\mathrm{N}_{\mathrm{i}}^{-1 / 3} \sim 20 \AA$ in a $5 \mathrm{~atm}$. gas. Thus impacts of the electron with nearest neighbors of its parent ion, as well as with the parent ion itself, can be expected to become frequent under these conditions. Mrreover, the quiver energy $(-300 \mathrm{eV})$ is at a value at which impact ionization cross sections are maximum.

Collisionless ionization of noninteracting atoms can be quantitatively modeled using an appropriate strong-field tunneling theory. A recent analysis of ion yield data in the rare gases by Augst et al. ${ }^{10}$ has shown that the formula of Ammosov 9 describes strong-field ionization of the rare gases in our intensity range reasonably accurately; the agreement with data improves as atomic number increases. Accordingly, the Ammosov formula has been used in modelling the ionization rate, but we also compare model results based on a Keldysh formula ${ }^{8}$. From the Ammosov theory, the probability per unit time $P_{i}$ for an atom to change from $i-$ to $(i+1)$-times ionized is calculated in terms of the ionization potential and the optical field strength. As in ion yield analysis, the role of $\mathrm{AC}$ Stark-shifted intermediate resonance states is neglected because, with the possible exception of the peak of the pulse ${ }^{11}$, any resonances are too transient to significantly affect ionization rate 35 . The time evolution of the density $N_{i}$ of ions in a particular ionization state ' $i$ ' is then determined from the $P_{i}$ through a series of coupled differential equations of the following form: 


$$
\frac{d N_{i}}{d t}=\left(P_{i-1} N_{i-1}-P_{i} N_{i}\right)
$$

The density of free electrons $N_{e}(t)$ can then be found in terms of the ion densities $N_{i}(t)$ by the following equation:

$$
N_{e}(t)=\sum_{i=0}^{h} i N_{i}(t)
$$

where ' $h$ ' is the ionization state of the atom. The rate of ionization at each of a sequence of time steps within the light pulse is calculated from Eq. (8) assuming a quasi-static instantaneous intensity, then convolved with the pulse intensity envelope. A RungeKutta scheme is employed to solve the differential equation for the populations of the various ionization states as a function of time. The density of free electrons is then used in eqn (1) to calculate the index of refraction as a function of time, and the resulting phase modulation and blueshift induced on the laser pulse is found according to eqn (2).

Figure 12a) shows calculated electron density $\mathrm{N}_{\mathrm{e}}(\mathrm{t})$ for $\mathrm{He}, \mathrm{Ar}$ and $\mathrm{Kr}$ gases (solid curves) for a $100 \mathrm{fs}$. pulse with peak intensity $10^{16} \mathrm{~W} / \mathrm{cm}^{2}$ using the Ammosov formula. $\mathrm{N}_{\mathrm{e}}(\mathrm{t})$ calculated with the same pulse parameters using the Keldysh formula, is shown (dashed cirves) for comparison. The ionizing pulse profile (dotted curve) is also shown for reference. Although the Ammosov formulation yields higher ionization rates (implying larger spectral shifts) than the Keldysh formulation, both formulations show ionization beginning very early in the pulse, then saturating near the peak of the pulse in each gas. Consequently only the first half of the pulse will become blueshifted according to this model; the trailing part of the pulse will remain unshifted, resulting in a spectral "shoulder" on the red side of the calculated spectra.

To calculate the shifted spectra, $\mathrm{N}_{\mathrm{e}}(\mathrm{t})$ must be computed for a spatially varying peak intensity along the propagation direction, converted to an index modulation via Eq. 
(1), then convolved with the transverse intensity profile of the ionizing pulse through the interaction region. We have performed such calculations using realistic models of the transverse intensity profile. Nevertheless, the choice of quantitative details in these models is very uncertain because defocusing introduces pressure-, intensity-, and speciesdependent variations in the transverse profile through the interaction region. On the other hand, we have also performed the calculations using a simplified cylindrical interaction region with a constant trarisverse intensity. In these simplified calculations, the product of peak intensity $x$ interaction length can be varied to fit the averaged magnitude of the blueshift for each measurement. In general, a range of peak intensity / interaction length products yield the same overall shift, and thus constitute an "isoshift" curve. We find that the same qualitative spectral features are reproduced not only along a substantial region of the isoshift curve in the simplified calculations, but also in calculations using a more complex focal geometry, although widths and amplitudes of spectral peaks vary. Thus the simplified calculations appear to illustrate the essential physics of ionization with fewer arbitrary assumptions, and have therefore been chosen for presentation in the following discussion. We caution that quantitative details of the focal geometry and peak intensity cannot be derived from our experiment. However, certain conclusions regarding the shape of the blueshifted spectrum, its time development, and its relation to time-varying ionization rate, appear to be independent of these quantitative details. Only such geometry-independent conclusions are drawn in the following discussion.

As an illustration, Fig. 12 b) shows self-shifted spectra calculated from the Ammosov $N_{e}(t)$ curves shown in Fig. 12 a) for peak intensity $10^{16} \mathrm{~W} / \mathrm{cm}^{2}$ and interaction length $7 \mu \mathrm{m}$. While quantitative details depend on the assumed interaction geometry, the blueshifted position of the spectral peak, and the prominent shoulder of unshifted light on the long wavelength side, are common to a wide range of assurned geometries. This model, using only tunneling ionization, appears adequate for describing 
the qualitative aspects of the blue shifting observed in $\mathrm{He}$ and $\mathrm{Ne}$ gas breakdown. However, calculated blueshifted spectra for the heavier gases $\mathrm{Ar}, \mathrm{Kr}, \mathrm{N}_{2}$, and $\mathrm{Xe}$ also display a large amount of unshifted light, inconsistent with the data. Another ionization process which occurs during the peak of the ionizing pulse and for a significant time afterwards is therefore necessary to account for the observed spectra in the heavier gases. This conclusion is independent of the assumed interaction geometry.

The process of ionization by impact with a quivering electron provides the proper qualitative behavior to complete the ionization model. The impact ionization rate is $N_{e} \sigma_{i} v_{e}\left(N_{e}=\right.$ density of free electrons, $v_{e}(t)=$ electron velocity, $\sigma_{i}\left(v_{e}\right)=$ cross section for impact ionization), and is included in the model by adding a pair of collisional terms to each of the coupled Eqs. (8):

$$
\frac{d N_{i}}{d t}=\left(P_{i-1} N_{i-1}-P_{i} N_{i}\right)+\left(N_{e} \sigma_{i-1} v_{e} N_{i-1}-N_{e} \sigma_{i} v_{e} N_{i}\right)
$$

and by coupling another equation to those above,

$$
\frac{d}{d t} v_{e}^{2}=\frac{d}{d t}\left(v_{t h}^{2}+v_{q u i v}^{2}\right)
$$

The last equation represents the transfer of energy from the light field to the ordered quiver motion of the electrons ( $v_{\text {quiv }}=$ quiver velocity), to random thermal motion in the plasma $\left(v_{t h}=\text { thermal velocity }\right)^{7}$. The impact ionization rate is highest near the peak of the $10^{16} \mathrm{~W} / \mathrm{cm}^{2}$ laser pulse, for three reasons: 1) in the noble gases, the cross sections $\sigma_{i}\left(E_{e}\right)$ reach a maximum for electron energy in the range $100 \mathrm{eV}<\mathrm{E}_{\mathrm{e}}<500 \mathrm{eV}$, corresponding closely to the quiver energy at this peak intensity 36 ; 2) $\mathrm{N}_{e}$ is large because of strong-field ionization earlier in the pulse; and 3 ) the average velocity $v_{e}$ of the free electrons reaches a maximum at the peak of the pulse. Nevertheless, in order for impact ionization to contribute significantly to the total ionization rate, the characteristic 
Femtosecond (Growth Dynamics..., Wood, Siders, Downer

time $\tau=\left(\mathrm{N}_{\mathrm{e}} \sigma_{\mathrm{i}} \mathrm{v}_{\mathrm{e}}\right)^{-1}$ must be smaller than the pulse duration. As an example, the time $\tau$ for a typical impact ionization process such as $\left(\mathrm{A}^{+}+\mathrm{e}^{-} \rightarrow \mathrm{A}^{2+}+2 \mathrm{e}^{-}\right)$can be estimated in 5 atm. of gas "A." $\mathrm{N}_{\mathrm{e}}-1.5 \times 10^{26} \mathrm{~m}^{-3}$, assuming complete single ionization of the 5 atm. gas. The r.m.s. value of $v_{e}$ is $1.0 \times 10^{7} \mathrm{~m} / \mathrm{s}$ at $10^{16} \mathrm{~W} / \mathrm{cm}^{2}$ (thermal contributions to $\mathrm{v}_{\mathrm{e}}$ are much smaller for plasma temperatures $\mathrm{kT}_{\mathrm{e}}<100 \mathrm{eV}$, as is the case in these experiments.) For ground state $\mathrm{He}^{+}, \sigma_{\mathrm{i}}-5.0 \times 10^{-18} \mathrm{~cm}^{2}$, yielding $\tau>1 \mathrm{ps}$, significantly longer than the pulse duration. For ground state $\mathrm{Ar}^{+}$, on the other hand, $\sigma_{\mathrm{i}}-1.2 \times 10^{-16}$ $\mathrm{cm}^{2}$, yielding $\tau \sim 50 \mathrm{fs}$, implying that impact ionization of $\mathrm{Ar}^{+}$would be likely to occur during the pulse. In general, $\sigma_{\mathrm{i}}$ increases with atomic number in the rare gas series, so for $\mathrm{Xe}$ and $\mathrm{Kr}$ gases, the times are even shorter. Such straightforward estimates therefore provide the rationale for invoking impact ionization.

For more precise calculations, however, considerable care is required in choosing the cross section $\sigma_{i}\left(E_{e}\right)$ values which are relevant to our experiment. As a first approximation, the above estimates used extensively tabulated experimental values, based on measurements of ion collection from e-beam excited gases ${ }^{36}$, or equivalently, values calculated from empirical formulae designed to fit this experimental data ${ }^{37}$. The form of the cross section as a function of electron energy is shown in fig. 13a) 36,37 . However, these $\sigma_{i}\left(E_{e}\right)$ values describe the impact ionization of electronic ground state atoms or ions, whereas the ions in our experiment, being dressed in an intense light field up to $10^{16} \mathrm{~W} / \mathrm{cm}^{2}$, are likely to be in highly excited electronic states when impact ionization becomes most important, namely at the peak of the pulse. Indeed, a recent experiment has shown evidence of large excited state populations remaining after short pulse ionization of rare gases ${ }^{38}$. In addition, recent dressed atom calculations of differential cross sections for $(e, 2 e)$ electron-impact ionization processes in atomic hydrogen support the idea that such processes will be significantly enhanced in strong light fields ${ }^{39}$. Consequently, some enhancement of $\sigma_{i}\left(E_{e}\right)$ is expected. Estimating the degree of enhancement is rendered difficult both by the lack of theoretical tools for 
estimating the excited state distribution and by the lack of experimental measurements of $\sigma_{i}\left(E_{e}\right)$ for excited state species. Nevertheless, Lotz recognized long ago that the $\sigma_{i}\left(E_{e}\right)$ of highly excited species could be an order of magnitude or more larger than the cross section of the corresponding ground state species ${ }^{37}$. Furthermore, Lotz pointed out that his (now widely used) empirical formulae for ground state species could be extended to calculate $\sigma_{i}\left(E_{e}\right)$ for particular electronic excited states of a parent atom or ion. Although the experimental excited state distribution may be highly uncertain, such calculations can at least provide guidance in estimating the range of $\sigma_{i}\left(E_{e}\right)$ values which are physically reasonable to consider in analyzing our experimental data. Accordingly, in Fig. 13b), results of Lotz formula calculations of $\sigma_{i}\left(\mathrm{E}_{\mathrm{e}}\right)$ for $\mathrm{Ar}^{2+}$ in the first few excited states are shown. The peak cross section nearly doubles when one electron is promoted to either of the first two excited states ( $3 \mathrm{~d}$ and $4 \mathrm{~s}$ ), and is 2.5 times larger when the electron begins in the $4 p$ excited state. As well, the threshold for impact ionization decreases. Similar calculations in each of the other noble gases reveals a similar magnitude increase of the peak impact ionization cross section. Larger enhancements are calculated for multiple electronic excitation. In view of these significant calculated enhancements of $\sigma_{i}\left(E_{e}\right)$, a sensible approach to modelling the data is to leave $\sigma_{i}\left(E_{e}\right)$ as an adjustable parameter within physically reasonable limits, with the ground state value of $c \quad j_{g}$ ) serving as a lower bound.

With these considerations, we now present quantitative calculations of plasma growth $\mathrm{N}_{e}(t)$ and blueshifting based on the combined tunneling/electron-impact ionization mechanisms described by Eq. (10). Fig. 14a) shows the calculated $N_{e}(t)$ in $\mathrm{He}, \mathrm{Ar}$ and $\mathrm{Kr}$ gases for a 100-fs laser pulse with gaussian temporal profile and peak intensity of $10^{16} \mathrm{~W} / \mathrm{cm}^{2}$, calculated using the Ammosov formula both with and without collisions at 1 and $5 \mathrm{~atm}$. pressure. The root-mean-squared velocity of an electron oscillating in the laser electric field is used for the collisional ionization. The tabulated values for the cross sections have been increased by a factor of 2.5 , based on comparison 
of the appearance of the calculated spectra with the data. Collisions begin to dominate the ionization process after approximately 3 or 4 electrons have been ionized, and the collisional process continues well beyond the peak of the ionizing pulse. This effect is least pronounced in $\mathrm{He}$ at $\mathrm{l}$ atm., and increases significantly both as pressure is increased, and as heavier gases are used. Figure 14b) shows calculated spectra using the density of free electrons found in 14a) in conjunction with the one-dimensional model for the focal region described above. Indeed, a much larger part of the pulse spectrum is shifted in Ar and $\mathrm{Kr}$ gases when collisional ionization is included, whereas the calculated spectrum for He is virtually unaffected. For the case of $\mathrm{Kr}$, Figure 14b) shows calculated spectra for both linearly and circularly polarized excitation. The somewhat higher tunneling ionization rate in the leading edge for circular polarization shows up as a slight enhancement of the more blueshifted peak. This slight polarization dependence is evidently too subtle to distinguish in the current experiments where the actual focal geometry is considerably more complicated then the cylindrical one assumed in the calculation. Similar slight polarization dependencies are calculated for other gases, and appear consistent with our observation of negligible polarization dependence.

To illustrate the effect of changes in laser pulse intensity, fig. 15 shows calculated spectral shifts in $5 \mathrm{~atm}$. Ar and $\mathrm{Kr}$ for a range of peak pulse intensities. Figures 15a) and b) show the calculated shifts for $\mathrm{Ar}$ and $\mathrm{Kr}$ for the same range of intensities as the experiment using the one-dimensional model without collisions. Both in Ar and $\mathrm{Kr}$, the model displays a shift of the spectral peak which increases with intensity, and eventually becomes a broad, blue shoulder. At the higher intensities a second peak is observed which remains near the original, unshifted, pulse spectrum. 15c) and d) show the results of including collisions in the model. Here, the results at the lower intensities are very similar to those obtained without collisions; at higher intensities, however, there is significantly less energy remaining near the original pulse spectrum. 
A more dramatic contrast is seen between the calculated time-resolved spectra from the model without collisions and the model including collisions.. Figs. 16a) and 16b) display calculated time-resolved spectra using the Ammosov model without collisions, and a 100 -fs. pulse with peak intensity $10^{16} \mathrm{~W} / \mathrm{cm}^{2}$. Figs. $16 \mathrm{c}$ ) and $16 \mathrm{~d}$ ) show calculated time-resolved spectra for the same pulse, with collisions included in the model. With the inclusion of collisions in both $\mathrm{Ar}$ and $\mathrm{Kr}$, it is seen that the pulse experiences significantly more shifting around $t=0$, and that the peak of the spectrum after $t=0$ remains shifted for a much longer time. This behaviour is compared in a more quantitative way in figure 17. 17a) and 17b) display plots of the temporal behaviour at $610 \mathrm{~nm}$ and $615 \mathrm{~nm}$ of the spectra in Ar and $\mathrm{Kr}$, respectively, for the model without collisions, and figs. 17c) and 17d) show the corresponding plots for the model with collisions included. (Spectral broadening and the rise at $t>200$ fs. in the model are artifacts of a finite array size in the program.) In both cases without collisions, the behaviour of the spectra at $610 \mathrm{~nm}$ correspond very well with measured data. However, the behaviour of the spectra at $615 \mathrm{~nm}$ in Ar displays a peak well before $\mathrm{t}=0$. In $\mathrm{Kr}, \mathrm{a}$ peak in the $615 \mathrm{~nm}$ temporal behaviour also exists before $t=0$, but is masked by a large dip as the bulk of the spectral energy is blue-shifted to shorter wavelengths. When collisions are included in the model, it is seen that in both $\mathrm{Ar}$ and $\mathrm{Kr}$ there is a peak in the temporal behaviour at $615 \mathrm{~nm}$ right at $\mathrm{t}=0$, significantly after the peak in the $610 \mathrm{~nm}$ behaviour. Thus, the model including the collisional ionization appears to yield a much better correspondence with the data.

The results of the modelling suggest that the two features in the measured spectra can be adequately explained by the two processes of strong-field ionization, and impact ionization. In general, the broad, blue shoulder on the shifted spectra arises from the primarily strong-field ionization early in the pulse, and the narrow, less-shifted peak can be ascribed to collisional ionization occurring during the peak of the pulse and immediately afterwards. Although collisions are not expected to play a significant role in 
$\mathrm{He}$ or $\mathrm{Ne}$, the two different rates of ionization observed in $\mathrm{He}$ and $\mathrm{Ne}$ at $\mathrm{l}$ atm. pressure, illustrated in figs. 10a) and 10b) can perhaps be attributed to the different rates of strong field ionization which occur early during the pulse in both of these gases. Fig. 14a) shows that the strong field ionization of both levels in He occur during a long enough time that the 100 -fs. probe can sample the process. This would suggest that experiments with shorter probing pulses and more sensitive measurements of the spectral shifts might reveal more details of the ionization dynamics, indicated by the $N_{e}(t)$ models in Figs. 12 and 14.

The two main conclusions which can be drawn from the experiment and model are that, under our intense field ionization conditions: 1 . There is a large blueshift (signifying large $\mathrm{dn} / \mathrm{dt}$ ) which saturates in the leading edge of the ionizing pulse, which is wellexplained by the Ammosov et al. theory of tunneling ionization. 2. There is an extra smaller blueshift (signifying small $\mathrm{dn} / \mathrm{dt}$ ) near the temporal peak of the ionizing pulse (not observed in the $\mathrm{He}$ and $\mathrm{Ne}$ data), which cannot be explained by a pure tunneling ionization mechanism. While we have shown that impact ionization in a strong light field explains the latter feature reasonably well - especially its occurrence at $\Delta t=0$, its absence in $\mathrm{He}$ and $\mathrm{Ne}$, and its approximate magnitude - we cannot rule out other nontunneling ionization mechanisms which might produce, or contribute to, the same effect. One interesting possibility is resonant enhancements of ionization cross section resulting from transient AC Stark shifts of excited ionic states into temporary resonance with a harmonic of the light field. Such resonances, first reported by Freeman et al. ${ }^{40}$, have recently been extensively observed ${ }^{11}$ in the photoelectron spectra of neutral rare gas atoms when multiphoton-ionized by ultrashort laser pulses. The narrow width of the observed photoelectron resonances has shown that such resonances are only effective near the temporal peak of the ionizing pulse, where they are slowly varying in time. Stark shifts in the leading or trailing edges of an ultrashort pulse cause excited state levels to sweep too rapidly through resonance to significantly enhance the ionization rate. 
Thus this mechanism appears consistent with extra ionization near $\Delta t=0$. Nevertheless, to our knowledge, such resonances have so far been observed only in first-stage ionization of neutral atoms at peak intensities lower than in our experiments. If present under our experimental conditions, such a mechanism would have to occur in severalfold ionized species which were tunnel-ionized early in the pulse. While an interesting possibility, theoretical tools anci supporting experiments are not yet available to warrant more detailed discussion.

\section{Summary}

We have presented a comprehensive set of blueshifting data of 100-femtosecond laser pulses caused by ionizing breakdown in $\mathrm{N}_{2}$ and in the noble gases $\mathrm{He}, \mathrm{Ne}, \mathrm{Ar}, \mathrm{Kr}$, and $\mathrm{Xe}$ at pressures ranging berween 1 and 5 atmospileres. Nonlinear polarization effects have been shown to play a negligible role in the modulation which causes the spectral changes, and also in the propagation of the pulse. Modelling on the phase modulation based soiely on strong-field ionization appears to adequately describe the qualitative features of the spectra arter breakdown in He and $\mathrm{Ne}$ gases. The data, however, reveal two distinct ionization rates in the heavier gases which are not well described by the strong-field theory alone: a fast rate of ionization which occurs early during the light pulse; and a slower rate, which occurs during the peak and latter half of the ionizing pulse. We have shown how the inclusion of a second, collisional, ionization process can provide a reasonable and adequate solution to this problem: early during the pulse, strong-field ionization occurs at a rapid rate. Near the peak of the pulse, the collisional process dominates the ionization, with an overall lower rate of ionization.

\section{Acknowledgments}

This research was supported by the Department of Energy (Grant DEFG05-92-ER40739), the National Science Foundation (Grant DMR8858388,) the Robert A. Welch 
Foundation (Grant F-1038,) and the Air Force Office of Scientific Research (Contract F49620-89-C-0044.)

\section{REFERENCES}

[1] N.H. Burnett and P.B. Corkum, "Cold-plasma production for recombination extreme-ultraviolet lasers by optical-field-induced ionization," J. Opt. Soc. Am. B, vol. 6, no. 6, pp. 1195-1199, June 1989.

[2] W.W. Jones and A.W. Ali, "Recombination lasers from cooled hydrogenlike plasmas," J. Appl. Phys, vol. 48, no. 7, p. 3118-3119, July 1977.

[3] J. Peyraud and N. Peyraud, "Population inversion in laser plasmas," J. Appl. Phys., vol. 43, no. 7, pp. 2993-2996, July 1972.

[4] T. Tajima and J.M. Dawson, "Laser electron accelerator," Phys. Rev. Lett., vol. 43, no. 4, pp. 267-270, July 1979.

[5] P. Sprangle, E. Esarey, A. Ting, and G. Joyce, "Laser wakefield acceleration and relativistic optical guiding," Appl. Phys. Lett., vol. 53, no. 22, pp. 2146-2148, November 1988.

[6] Wm M. Wood, G.B. Focht, M.C. Downer, "Tight focusing and blue shifting of millijoule femtosecond pulses from a conical axicon amplifier," Opt. Lett., vol. 13, no. 11, pp. 984-986, November 1988.

[7] B.M. Penetrante and J.N. Bardsley, "Residual energy in plasmas produced by intense subpicosecond lasers," Phys. Rev. A, vol. 43, no. 6, pp. 3100-3113, March 1991.

[8] L.V. Keldysh, "Ionization in the field of a strong electromagnetic wave," Sov. Phys. JETP, vol. 20, no. 5, pp. 1307-1314, May 1965.

[9] M.V. Ammosov, N.B. Delone, and V.P. Krainov, "Tunnel ionization of complex atoms and of atomic ions in an altemating electromagnetic field," Sov. Phys. JETP, vol. 64, no. 6, pp. 1191-1194, December 1986.

[10] S. Augst, D.D. Meyerhofer, D. Strickland, and S.L. Chin, "Laser ionization of noble gases by Coulomb-barrier suppression," J. Opt. Soc. Am. B, vol. 8, no. 4, pp. 858-867, April 1991.

[11] R.R. Freeman and P.H. Bucksbaum, "Investigations of above-threshold ionization using subpicosecond laser pulses," J. Phys. B, vol. 24, pp. 325-347, 1991. 
[12] E. Yablonovitch, "Self-phase modulation and short-pulse generation from laserbreakdown plasmas," Phys. Rev. A, vol. 10, no. 5, pp. 1888-1895, November 1974.

[13] Wm.M. Wood, C.W. Siders, and M.C. Downer, "Measurement of femtosecond ionization dynamics of atmospheric density gases by spectral blueshifting," Phys. Rev. Lett., vol. 67, no. 25, pp. 3523-3526, December 1991.

[14] V.I. Semenova, "Reflection of electromagnetic waves from an ionization front," Sov. Radiophys., vol 10, no. 8, pp. 599-604, 1967.

[15] M. Lampe, E. Ott, and J.H. Walker, "Interaction of electromagnetic waves with a moving ionization front," Phys. Fluids, vol. 21, no. 1, pp. 42-54, January 1978.

[16] Wm.M. Wood, Femtosecond Time-resolved Study of Plasma Generation and Dynamics by Blueshifting of High Intensity Laser Pulses, Ph.D. Dissertation, University of Texas at Austin, May 1991.

[17] M.C. Downer, W.M. Wood, and J.I. Trisnadi, "Comment on 'Energy conservation in the picosecond and subpicosecond photoelectric effect'," Phys. Rev. Lett., vol. 65, no. 22 , p. 2832 , November 1990.

[18] B.M. Penetrante, J.N. Bardsley, Wm.M. Wood, C.W. Siders, and M.C. Downer, "Ionization induced frequency shifts in intense femtosecond laser pulses," J. Opt. Soc. Am. B, vol. 9, no. 11, pp. 2032-2040, November 1992.

[19] S.C. Wilks, J.M. Dawson, and W.B. Mori, "Frequency up-conversion of electromagnetic radiation with use of an overdense plasma," Phys. Rev. Lett., vol. 61 , no. 3, pp. 337-340, July 1988.

[20] S.C. Wilks, J.M. Dawson, W.B. Mori, T. Katsouleas, and M.E. Jones, "Photon Accelerator," Phys. Rev. Lett., vol. 62, no. 22, pp. 2600-2603, May 1989.

[21] E. Esarey, G. Joyce, and P. Sprangle, "Frequency up-shifting of laser pulses by copropagating ionization fronts," Phys. Rev. A, vol. 44, no. 6, pp. 3908-3911, September 1991.

[22] W.B. Mori, "Generation of tunable radiation using an underdense ionization front," Phys. Rev. A, vol. 44, no. 8, pp. 5118-5121, October 1991.

[23] R.L. Savage Jr., C. Joshi, and W.B. Mori, "Frequency upconversion of electromagnetic radiation upon transmission into an ionization front," Phys. Rev. Lett., vol. 68, no. 7, pp. 946-949, February 1992.

[24] R.L. Savage et al., IEEE Trans. Plasma Science (this volume).

[25] E. Yablonovitch, "Energy conservation in the picosecond and subpicosecond photoelectric effect," Phys. Rev. Lett., vol. 60, no. 9, pp. 795-796, February 1988. 
Femtosecond (irowth Dynamics..., Wood, Siders, Downer

[26] E.P. Ippen and C.V. Shank, Ultrashort Light Pulses: Picosecond Techniques and Applications, ed. S.L. Shapiro, Berlin: Springer-Verlag, 1984, pp. 102-108.

[27] P.B. Corkum, C. Rolland, and T. Srinivasan-Rao, "Supercontinuum generation in gases," Phys. Rev. Lett., vol. 57, no. 18, pp. 2268-2271, November 1986.

[28] N. Bloembergen, Nonlinear Optics, London: W.A. Benjamin, 1965.

[29] J.W. Shearer and J.L. Eddleman, "Laser light forces and self-focusing in fully ionized plasmas," Phys. Fluids, vol. 16, no. 10, pp. 1753-1761, October 1973.

[30] J.F. Drake, P.K. Kaw, Y.C. Lee, G. Schmidt, C.S. Liu, and M.N. Rosenbluth, "Parametric instabilities of electromagnetic waves in plasmas," Phys. Fluids, vol. 17, no. 4, pp. 778-785, April 1974.

[31] D. L. Fisher, T. Tajima, and M. C. Downer, "Wakefield excitation by an ultrashort laser pulse through the ionization potential," Bull. Am. Phys. Soc., vol. 36, pp. 2364$2365,1991$.

[32] E.L. Dawes, "Optical third-harmonic coefficients for the inert gases," Phys. Rev., vol. 169 , no. 1 , pp. 47-48, May 1968.

[33] G.H.C. New and J.F. Ward, "Optical third-harmonic generation in gases," Phys. Rev. Lett., vol. 19, no. 10, pp. 556-559, September 1967.

[34] H.J. Lehmeier, W. Leupacher, and A. Penzkofer, "Nonresonant third order hyperpolarizability of rare gases and $\mathrm{N}_{2}$ determined by third harmonic generation," Opt. Comm., vol. 56, no. 1, pp. 67-72, November 1985.

[35] S.L. Chin, C. Rolland, P.B. Corkum, and P. Kelly, "Multiphoton ionization of Xe and $\mathrm{Kr}$ with intense $0.62 \mu \mathrm{m}$ femtosecond pulses," Phys. Rev. Lett., vol. 61, no. 2, pp. 153-156, July 1988.

[36] H.Tawara and T.Kato, "Total and partial ionization cross sections of atoms and ions by electron impact," Atomic Data and Nuclear Data Tables, vol. 36, pp. 167-353, 1987.

[37] W. Lotz, "An empirical formula for the electron-impact ionization cross-section," Zeit. F. Phys., vol. 206, pp. 205-211, 1967.

[38] M.P. de Boer and H.G. Muller, "Observation of large populations in excited states after short-pulse multiphoton ionization," Phys. Rev. Lett., vol. 68, no, 18, pp. 2747 2750, May 1992.

[39] P. Martin, V. Veniard, A. Maquet, P. Franken, and C.J. Joachain, "Electron-impact ionization of atomic hydrogen in the presence of a laser field," Phys. Rev. A, vol. 39, no. 12, pp. 6178-6189, June 1989. 
Femtosecond Growth Dynamics..., Wood, Siders, Downer

[40]. R. R. Freeman, P.H. Bucksbaum, H. Milchberg, S. Darack, D. Schumacher, and M. E. Geusic, "Above-threshold ionization with subpicosecond laser pulses," Phys. Rev. Lett., vol. 59, no. 10, pp. 1092-1095, September 1987. 
Femtosecond Growth Dynamics..., Wood, Siders, Downer

\section{Bio-sketches of authors}

Wm. Monty Wood was born in Walnut Creek, CA, in 1960. He received the Bachelor's degree in physics from Reed College, Portland, OR, in 1983 and the Ph.D. from the University of Texas at Austin in 1991. His dissertation concerned the femtosecond time-resolved study of plasma generation and dynamics by blueshifting of high intensity laser pulses.

Craig W. Siders was born in Columbus, $\mathrm{OH}$, in 1966. He received the Bachelor's degree in physics, with Highest Honors, from Kenyon College, Gambier, OH, in 1988. He then worked for Dow Chemical's Measurement Science Group in Granville, OH. In 1989, he enrolled in the University of Texas at Austin, where he is currently a Ph. D. candidate in physics. His current research interests are in the interaction of ultrafast, ultraintense laser pulses with gases and plasmas.

Mr. Siders is a member of Phi Beta Kappa, Sigma Xi, and Phi Kappa Phi.

M. C. Downer did his graduate research at Harvard University on nonlinear optical spectroscopy of lanthanide-doped crystals. After receiving a Ph.D. in applied physics in 1983, he did postdoctoral work at AT\&T Bell Laboratories, where he developed the technique of femtosecond strobe photography, and made numerous contributions to femtosecond spectroscopy of semiconductors and to development of femtosecond light sources. In 1985, he joined the faculty of the Physics Department at the University of Texas at Austin. His recent research has centered on extreme states of matter created by intense femtosecond irradiation, and on the optical probing of semiconductor surfaces. he received an IBM Faculty Development Award in 1987, and was named a Presidential Young Investigator and an Office of Naval Research Young Investigator in 1988 . He is currently Associate Professor of Physics. 
Femtosecond (Growth Dynamics..., $\because$ sod, Siders, Downer

\section{Tables}

\begin{tabular}{|c|c|c|c|c|c|}
\hline Gas: & $\mathrm{He}$ & $\mathrm{Ne}$ & $\mathrm{Ar}$ & $\mathrm{Kr}$ & $\mathrm{Xe}$ \\
\hline 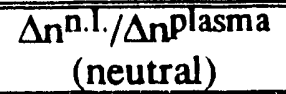 & $3.6 \times 10^{-3}$ & $6.6 \times 10^{-3}$ & 0.10 & 0.24 & 0.68 \\
\hline$(+1)$ & $7.7 \times 10^{-5}$ & $6.4 \times 10^{-4}$ & $8.6 \times 10^{-3}$ & $1.9 \times 10^{-2}$ & $4.2 \times 10^{-2}$ \\
\hline$(+2)$ & - & $6.0 \times 10^{-5}$ & $1.2 \times 10^{-3}$ & $2.2 \times 10^{-3}$ & $5.1 \times 10^{-3}$ \\
\hline
\end{tabular}

Table 1: Ratio of the calculated nonlinear index change of neutral atoms ( $2^{\text {nd }}$ row), singly ( $3^{\text {rd }}$ row), or doubly (4th row) ionized ion cores to the index change caused by a plasma consisting of a single free electron per ion at intensity $I=10^{16} \mathrm{~W} / \mathrm{cm}^{2}$.

\begin{tabular}{|l|c|c|c|c|c|c|c|}
\hline $\begin{array}{l}\text { Degree of } \\
\text { Ionization }\end{array}$ & $1 \mathrm{x}$ & $2 \mathrm{x}$ & $3 \mathrm{x}$ & $4 \mathrm{x}$ & $5 \mathrm{x}$ & $6 \mathrm{x}$ & $7 \mathrm{x}$ \\
\hline $\mathrm{He}$ & 0.084 & - & - & - & - & - & - \\
\hline $\mathrm{Ne}$ & 0.40 & 0.15 & 0.067 & 0.036 & 0.020 & 0.010 & 0.004 \\
\hline $\mathrm{Ar}$ & 1.0 & 0.39 & 0.19 & 0.11 & 0.058 & 0.028 & 0.012 \\
\hline $\mathrm{Kr}$ & 1.31 & 0.51 & 0.26 & 0.15 & 0.082 & 0.039 & 0.019 \\
\hline $\mathrm{Xe}$ & 1.69 & 0.65 & 0.33 & 0.19 & 0.11 & 0.058 & 0.029 \\
\hline
\end{tabular}

Table 2: Ratio of the maximum index change from bound electrons to the index change from a plasma. 


\section{Figure Captions}

Figure 1: Experimental set-up for the single-pulse and time-resolved investigations of the blue shift. The single-pulse experiments are done without the beamsplitter $A$, and with the polarizing cubes B and C replaced by neutral density filters whose sum is constant. Time-resolved experiments utilize a probing beam containing $4 \%$ of the pump beam energy split off with beamsplitter $\mathbf{A}$, sent down a retroreflecting delay arm, and recombined with the pump pulse after being orthogonally polarized.

Figure 2: a) Autocorrelation traces for $A$, the amplified pulse; B, the amplified, attenuated pulse traversing the experiment with no breakdown; and $C$, the spectrally shifted pulse after ionizing breakdown. b) Laser pulse spectrum from the amplifier, befire ionizing breakdown.

Figure 3: Spectral shifting data for single pulses as a function of pulse peak intensity, at 1 atmosphere pressure. Peak intensity increases towards the bottom of each figure, with a) helium ranging between $4 \times 10^{14} \mathrm{~W} / \mathrm{cm}^{2}$ and $10^{16} \mathrm{~W} / \mathrm{cm}^{2}$, b) through e) ranging between $10^{14} \mathrm{~W} / \mathrm{cm}^{2}$ and $10^{16} \mathrm{~W} / \mathrm{cm}^{2}$, and $\mathrm{f}$ ) xenon ranging between $2.5 \times 10^{13} \mathrm{~W} / \mathrm{cm}^{2}$ and $10^{16} \mathrm{~W} / \mathrm{cm}^{2}$. The peak intensity at which a recombination spark is first visible is indicated by a heavy horizontal bar. The vertical bar indicates the center of the unshifted spectrum.

Figure 4: Spectral shifting data for single pulses as a function of pulse peak intensity, at 5 atmospheres pressure. Peak intensity increases towards the bottom of each figure, with a) helium ranging between $4 \times 10^{14} \mathrm{~W} / \mathrm{cm}^{2}$ and $10^{16} \mathrm{~W} / \mathrm{cm}^{2}$, b) through e) ranging between $10^{14} \mathrm{~W} / \mathrm{cm}^{2}$ and $10^{16} \mathrm{~W} / \mathrm{cm}^{2}$, and $\mathrm{f}$ ) xenon ranging between $2.5 \times 10^{13} \mathrm{~W} / \mathrm{cm}^{2}$ 
and $10^{16} \mathrm{~W} / \mathrm{cm}^{2}$. The peak intensity at which a recombination spark is first visible is indicated by a heavy horizontal bar. The vertical bar indicates the center of the unshifted spectrum.

Figure 4: Spectral shifting data for single pulses of peak intensity $10^{16} \mathrm{~W} / \mathrm{cm}^{2}$ as a function of gas pressure. Pressure increases towards the bottom of the figures, and ranges between 1 - and 5- atmospheres. The vertical bar indicates the center of the unshifted spectrum.

Figure 5: Spectral shifting data for single pulses with peak intensity $10^{16} \mathrm{~W} / \mathrm{cm}^{2}$ as a function of gas species, for 1- and 5- atmospheres pressure. Noble gas atomic weight increases towards the bottom of the figures, with nitrogen inserted between argon and krypton. The vertical bar indicates the center of the unshifted spectrum.

Figure Error! Bookmark not defined.: Autocorrelation measurement for the pulses used in the time-resolved experiments.

Figure 8: Time-resolved probe spectra in each of the gases in 1 atmosphere pressure, using peak pump pulse intensity of $10^{16} \mathrm{~W} / \mathrm{cm}^{2}$. Time ranges between $-640 \mathrm{fs}$. (probe arriving before the pump pulse) to $480 \mathrm{fs}$. (probe arriving after the pump pulse.) Time $t=0$, corresponding to the overlap of the peaks of the pump and the probe pulses, is marked by an emboldened spectrum.

Figure 9: Time-resolved probe spectra in each of the gases in 5 atmosphere pressure, using peak pump pulse intensity of $10^{16} \mathrm{~W} / \mathrm{cm}^{2}$. Time ranges between $-640 \mathrm{fs}$. (probe arriving before the pump pulse) to $480 \mathrm{fs}$. (probe arriving after the pump pulse.) Time 
$t=0$, corresponding to the overlap of the peaks of the pump and the probe pulses, is marked by an emboldened spectrum.

Figure 10: Temporal behaviour of those portions of the spectra around $610 \mathrm{~nm}$ and $615 \mathrm{~nm}$ for each of the gases at 1 atmosphere pressure.

Figure 11: Temporal behaviour of those portions of the spectra around $610 \mathrm{~nm}$ and $615 \mathrm{~nm}$ for each of the gases at 5 atmospheres pressure.

Figure 12: Degree of ionization as a function of time predicted from the model, without collisional ionization, using pulse peak intensity of $10^{16} \mathrm{~W} / \mathrm{cm}^{2}$. The ionizing pulse temporal profile is shown for reference. a) compares results from the Keldysh and Ammosov formulations in $\mathrm{He}, \mathrm{Ar}$ and $\mathrm{Kr}$ gases. b) shows calculated spectra using the Ammosov formulation.

Figure 13: a) Functional form for the collisional ionization cross-sections of atom "A": $\left(A^{+}+e^{-} \rightarrow A^{++}+2 e^{-}\right)$as a function of electron energy for each of the noble gases. b) Increase of cross section predicted by Lotz for $\mathrm{Ar}^{++}$in the three lowest excited states.

Figure 14: Degree of ionization as a function of time calculated from the model including collisional ionization, using pulse peak intensity of $10^{16} \mathrm{~W} / \mathrm{cm}^{2}$. The ionizing pulse profile is shown for reference. a) compares results from the Ammosov formulation with and without collisions in $\mathrm{He}, \mathrm{Ar}$ and $\mathrm{Kr}$ gases at $5 \mathrm{~atm}$. pressure. b) shows the calculated shifts when collisions are included. 
Femtosecond Growth Dynamics.... Wood, Siders, Downer

Figure 15: Calculated blueshifted single pulse spectra in 5 atmosphere pressure Ar and $\mathrm{Kr}$ gases using pulse peak intensities in the range $10^{14} \mathrm{~W} / \mathrm{cm}^{2}$ to $10^{16} \mathrm{~W} / \mathrm{cm}^{2}$, comparing the Ammosov model without collisions, a) and b), and with collisions, c) and d).

Figure 16: Calculated time-resolved blueshifted pulse spectra in 5 atmosphere pressure Ar and $\mathrm{Kr}$ gases using pulse peak intensity of $10^{16} \mathrm{~W} / \mathrm{cm}^{2}$, comparing the Ammosov model without collisions, a) and b), and with collisions, c) and d).

Figure 17: Calculated temporal behaviour of those portions of the laser spectra at 610 $\mathrm{nm}$ and $615 \mathrm{~nm}$ in $5 \mathrm{~atm}$. Ar and $\mathrm{Kr}$ gases using pulse peak intensity of $10^{16} \mathrm{~W} / \mathrm{cm}^{2}$, comparing the Ammosov model without collisions, a) and b), and with collisions, c) and d). 
Femtosecond Growth Dynamics..., Wood, Siders, Downer

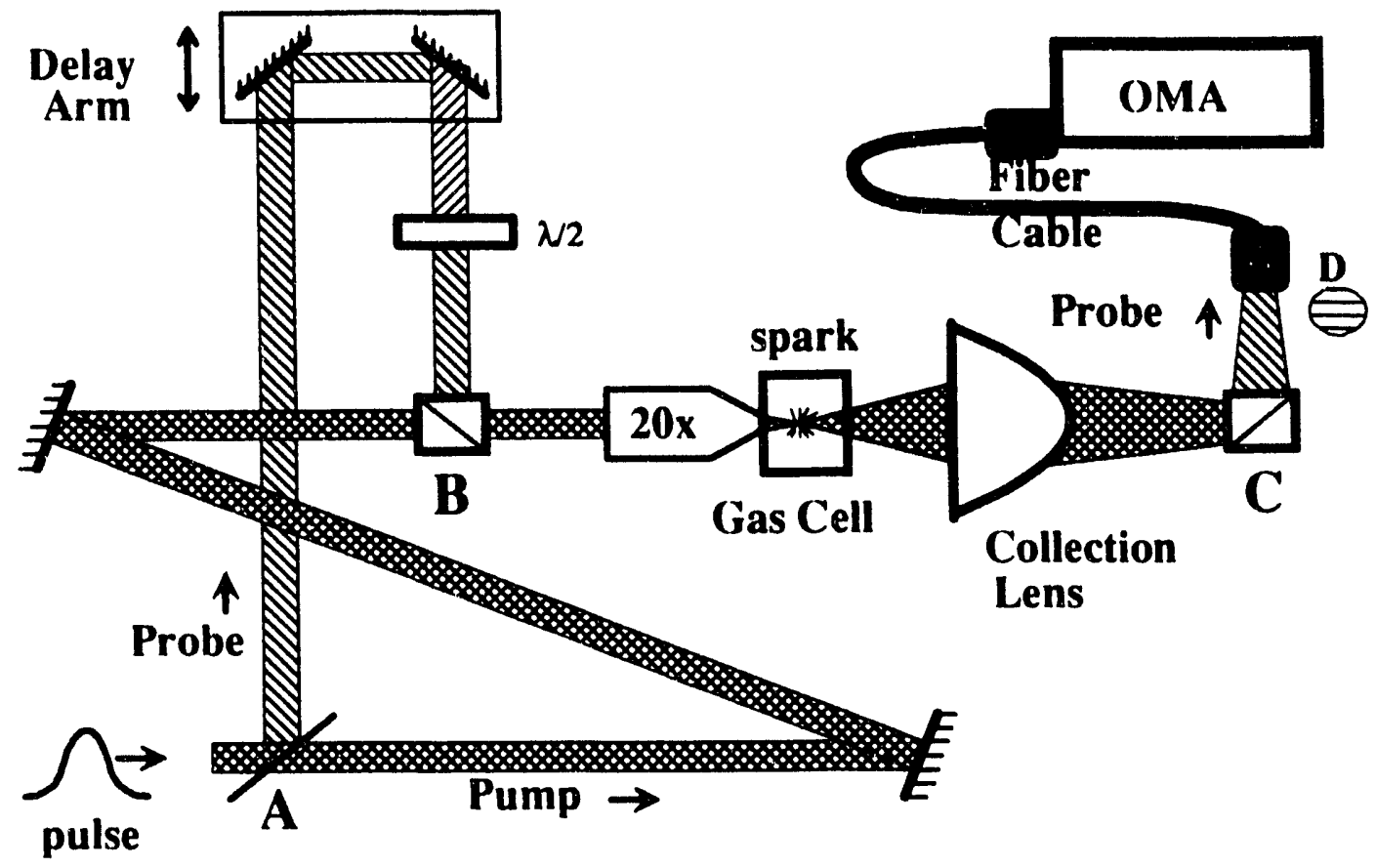


Femtosecond Growtin Dynamics..., Wood, Siders, Downer
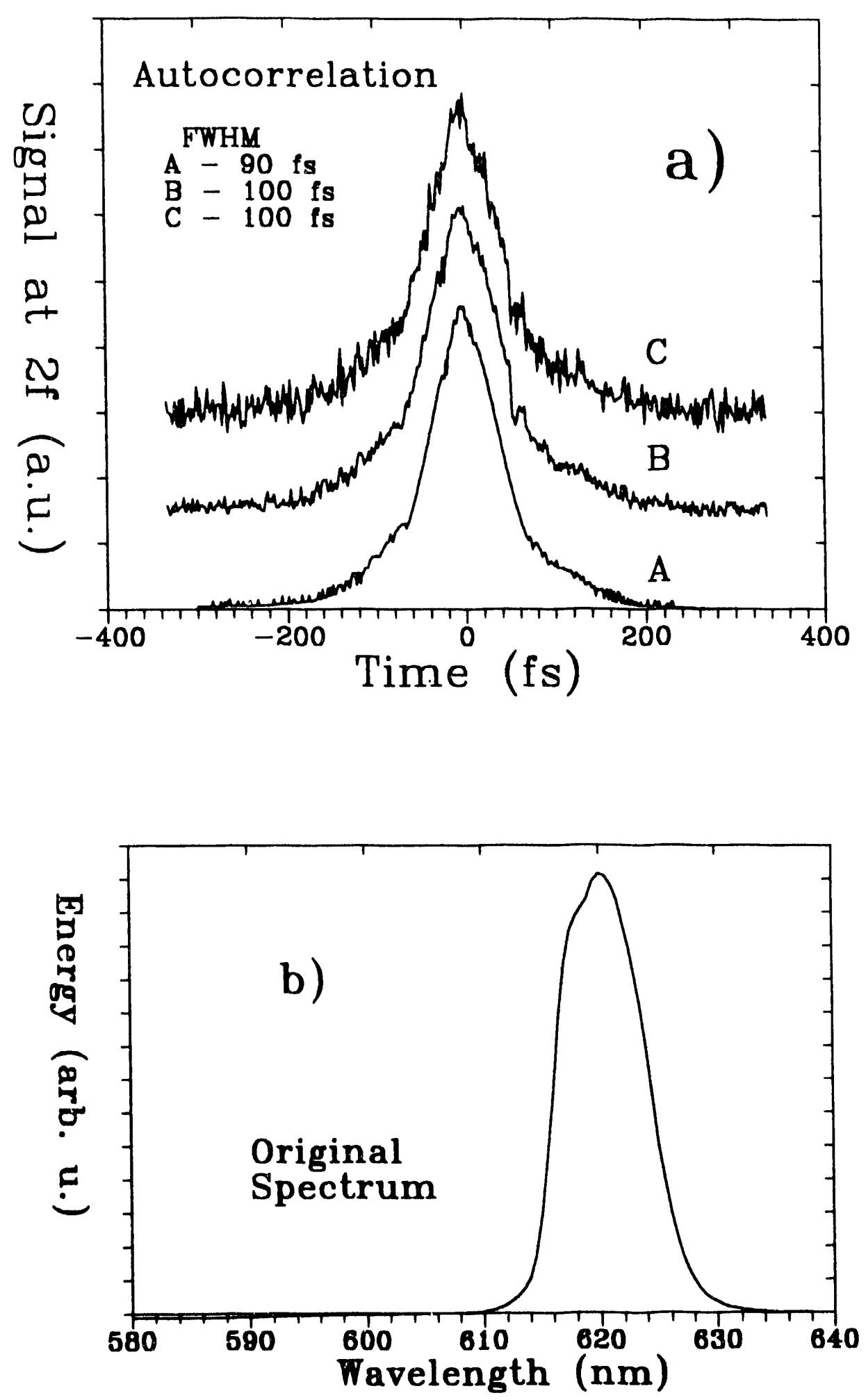
Femtosecond Growth Dynamics..., Wood, Siders, Downer
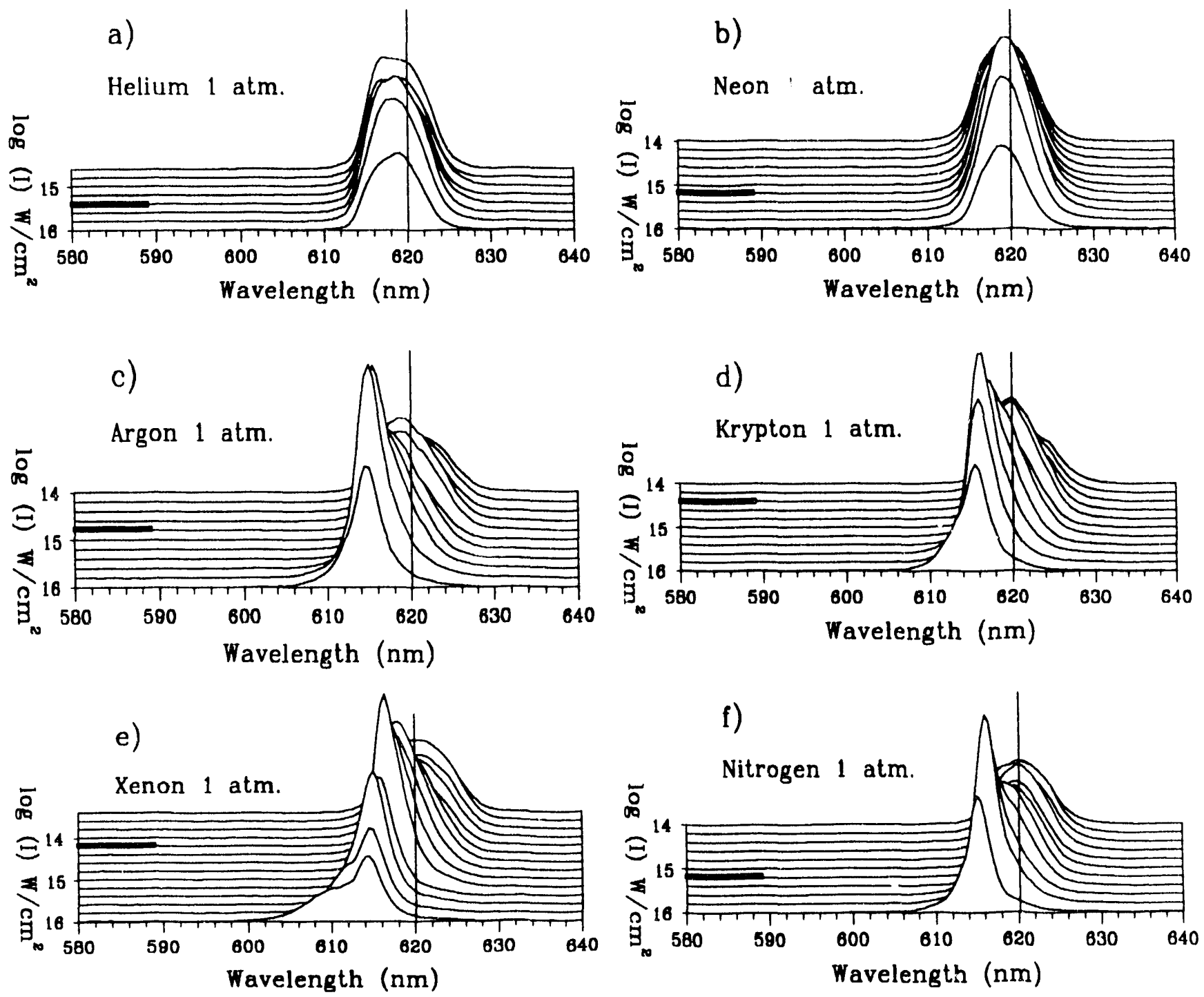

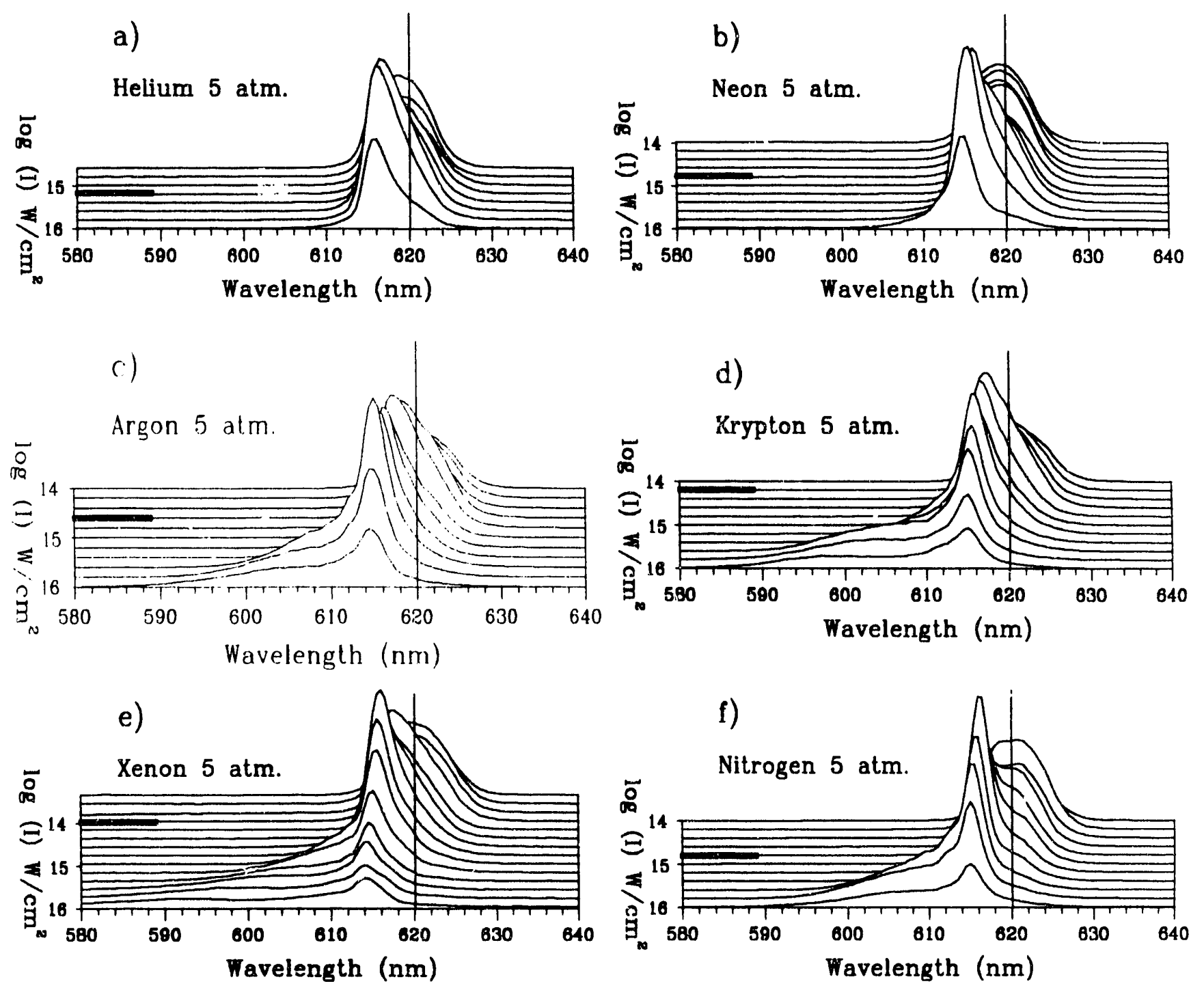
Femtosecond Growth Dynamics..., Wood, Siders, Downer
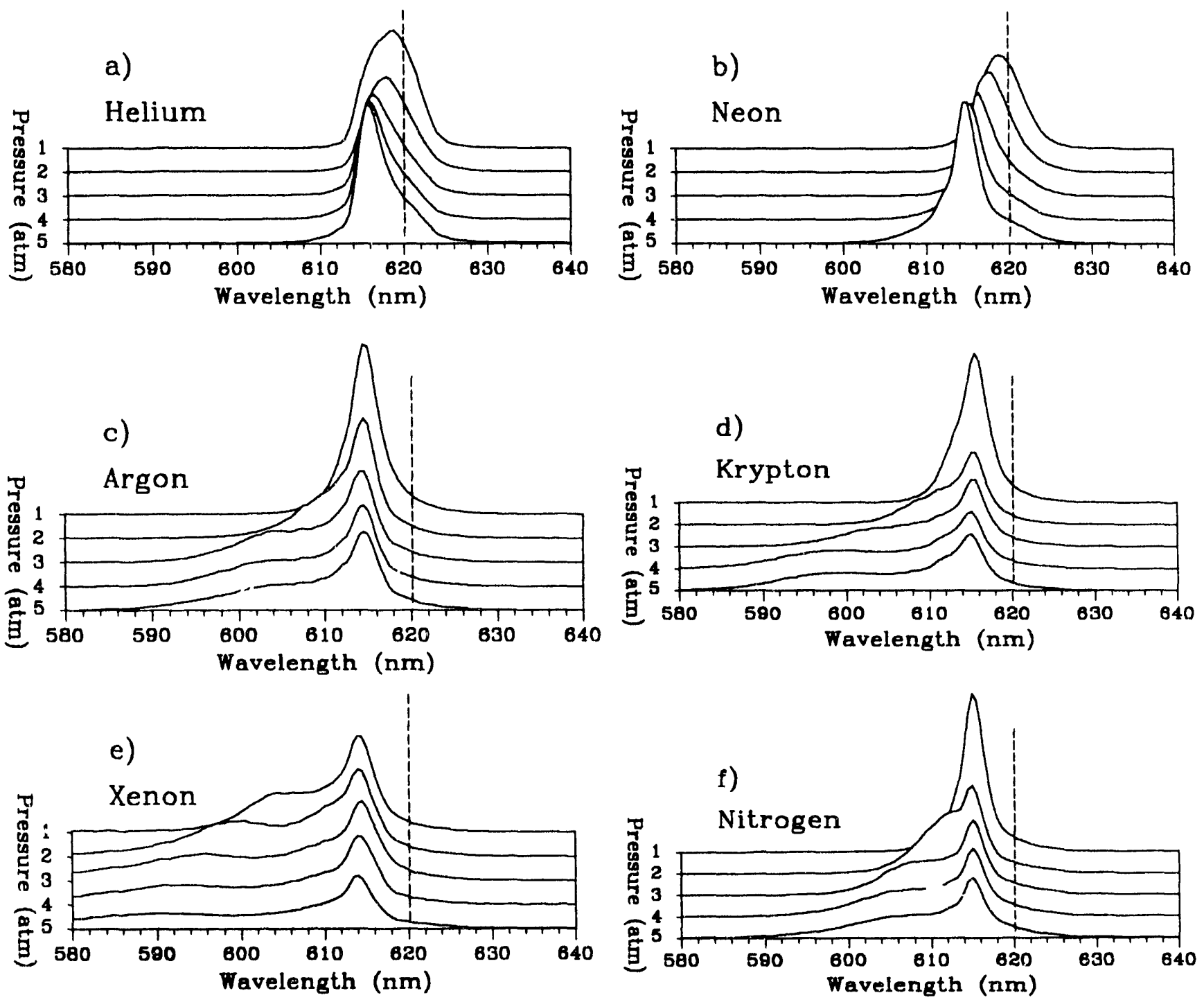
Femtosecond Growth Dynamics..., Wood, Siders, Downer

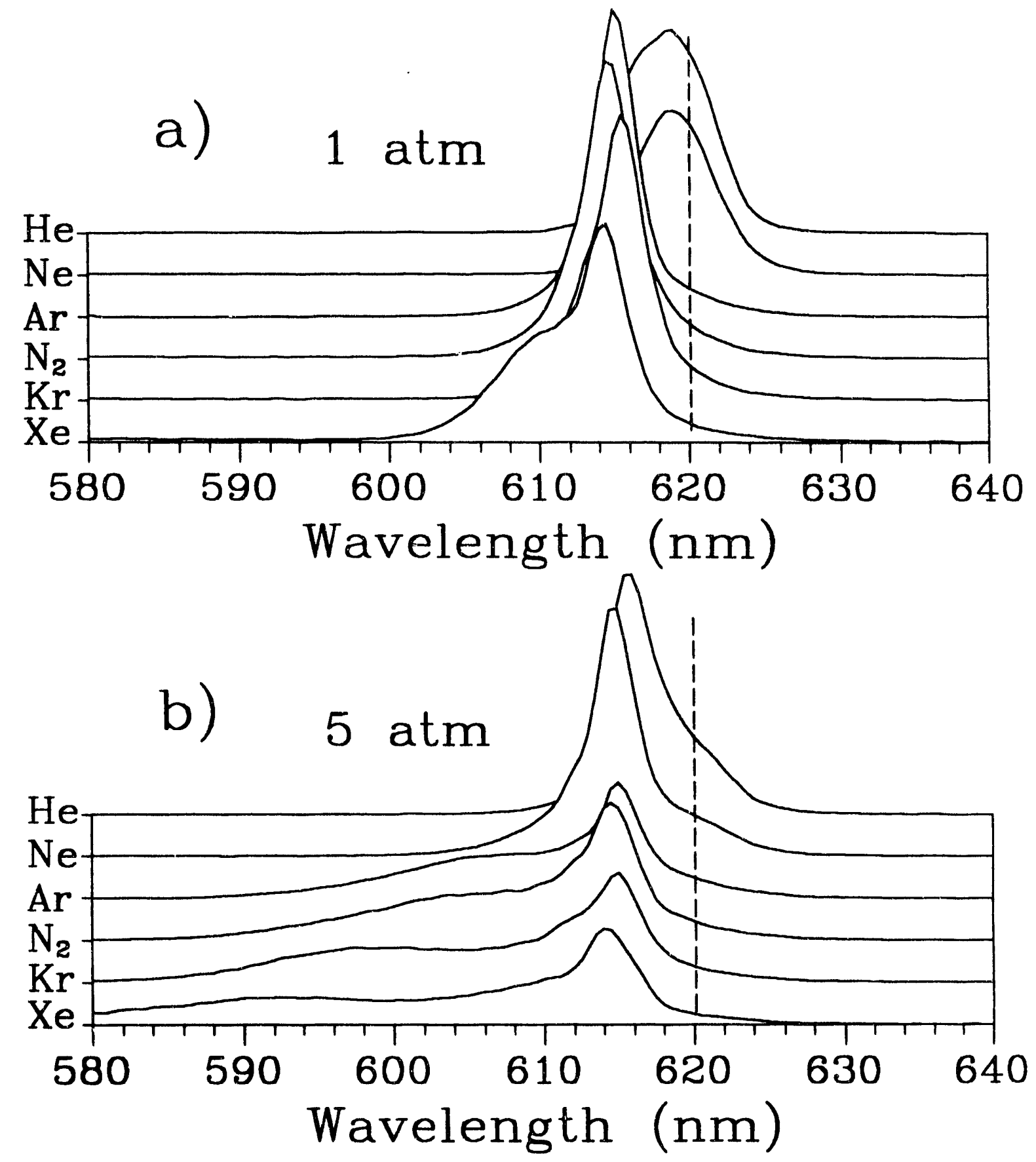


Femtosecond Growth Dynamics..., Wood, Siders, Downer

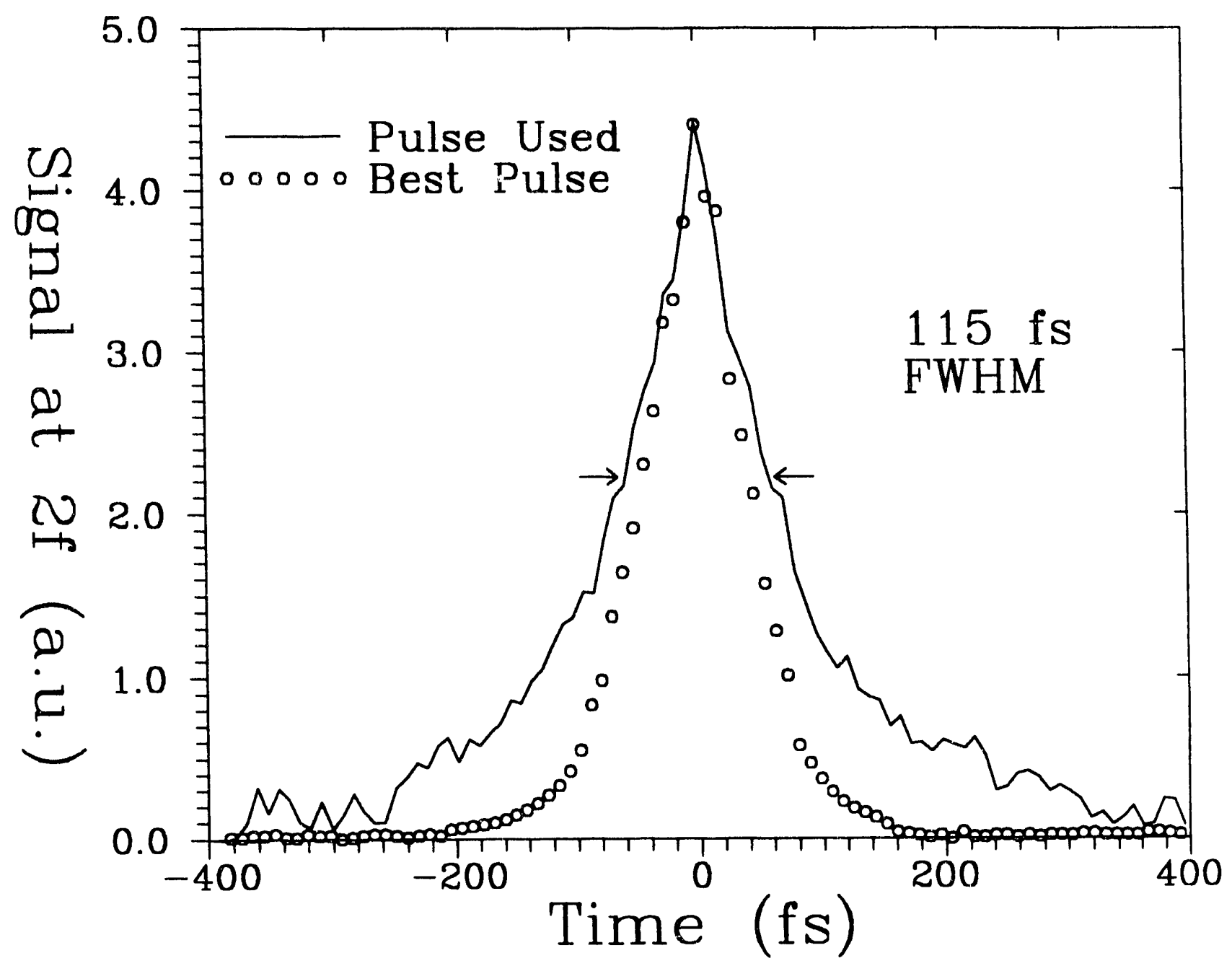


Femtosecond Growth Dynamics..., Wood, Siders, Downer
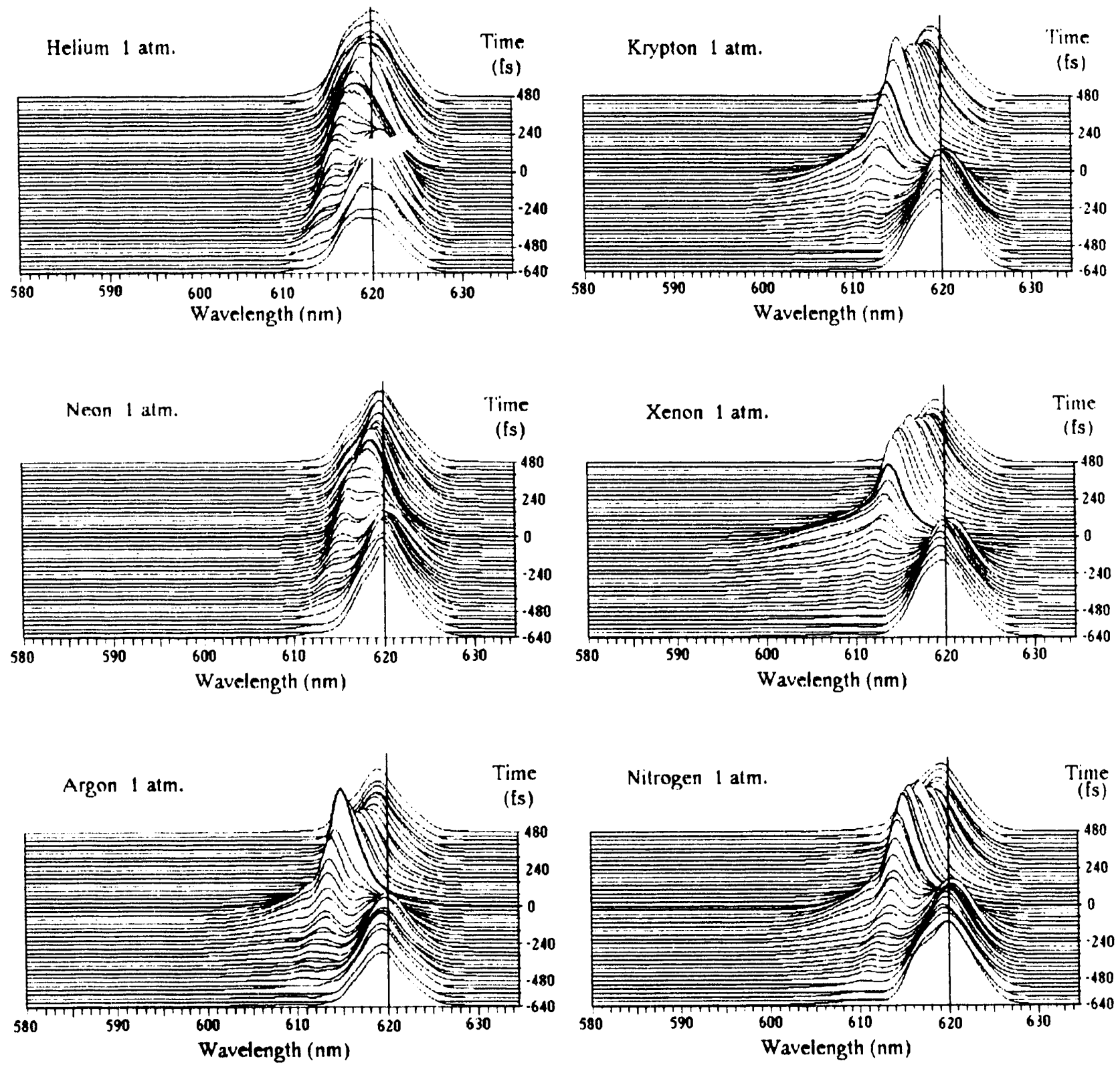
Femtosecond Growth Dynamics..., Wood, Siders, Downer
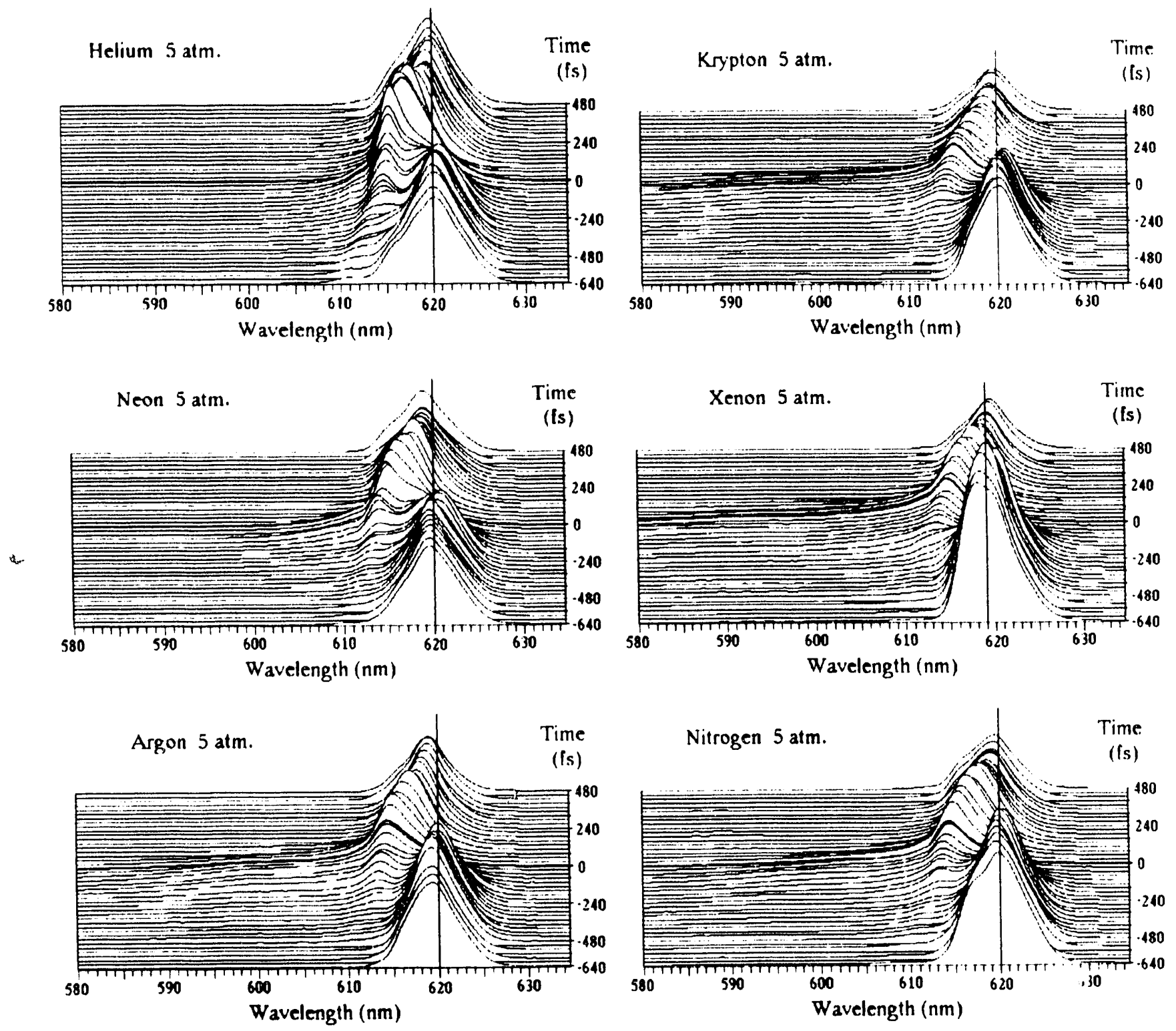
Femtosecond Growth Dynamics..., Wood, Siders, Downer
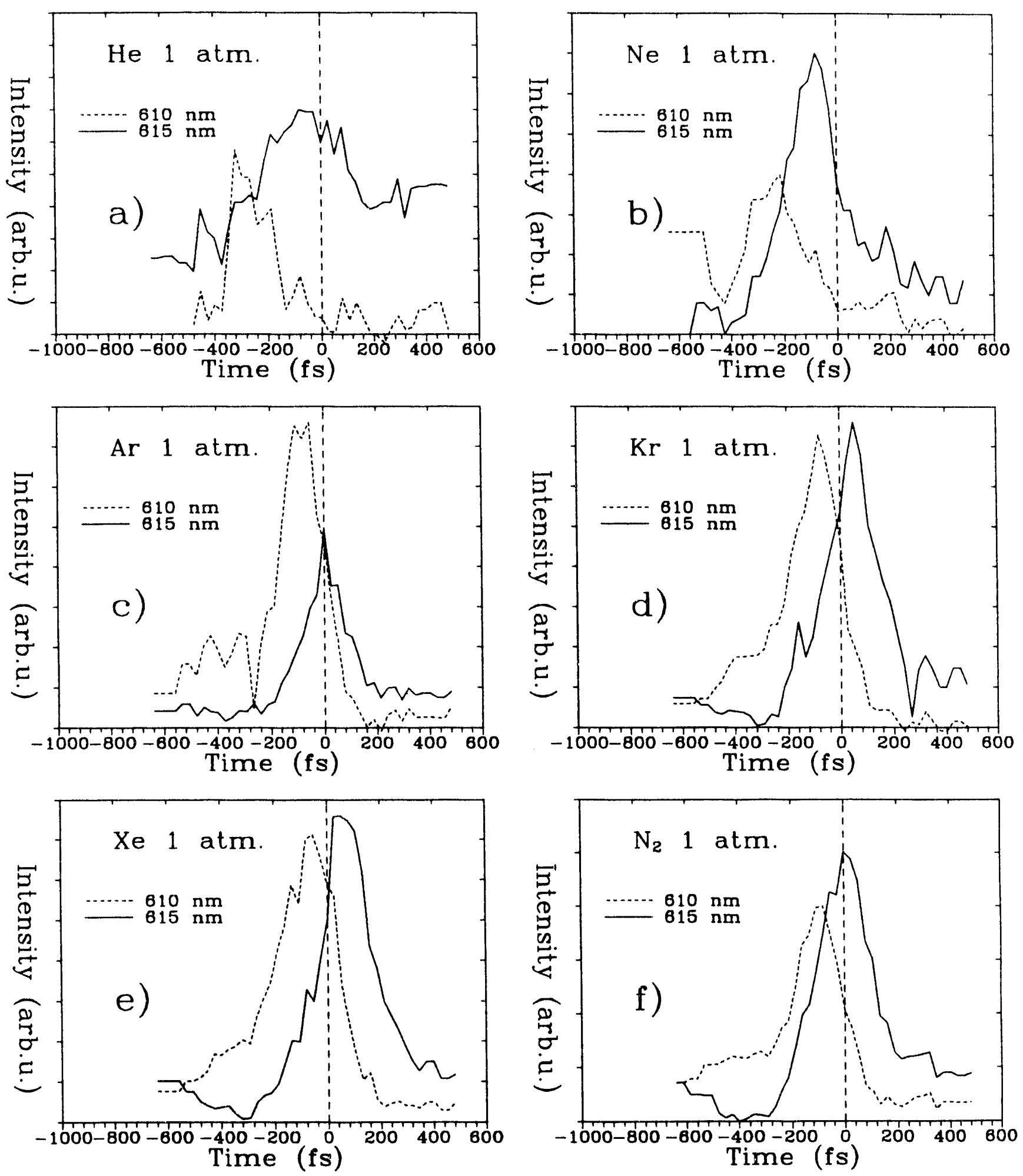
Femtosecond Growth Dynamics..., Wood, Siders, Downer
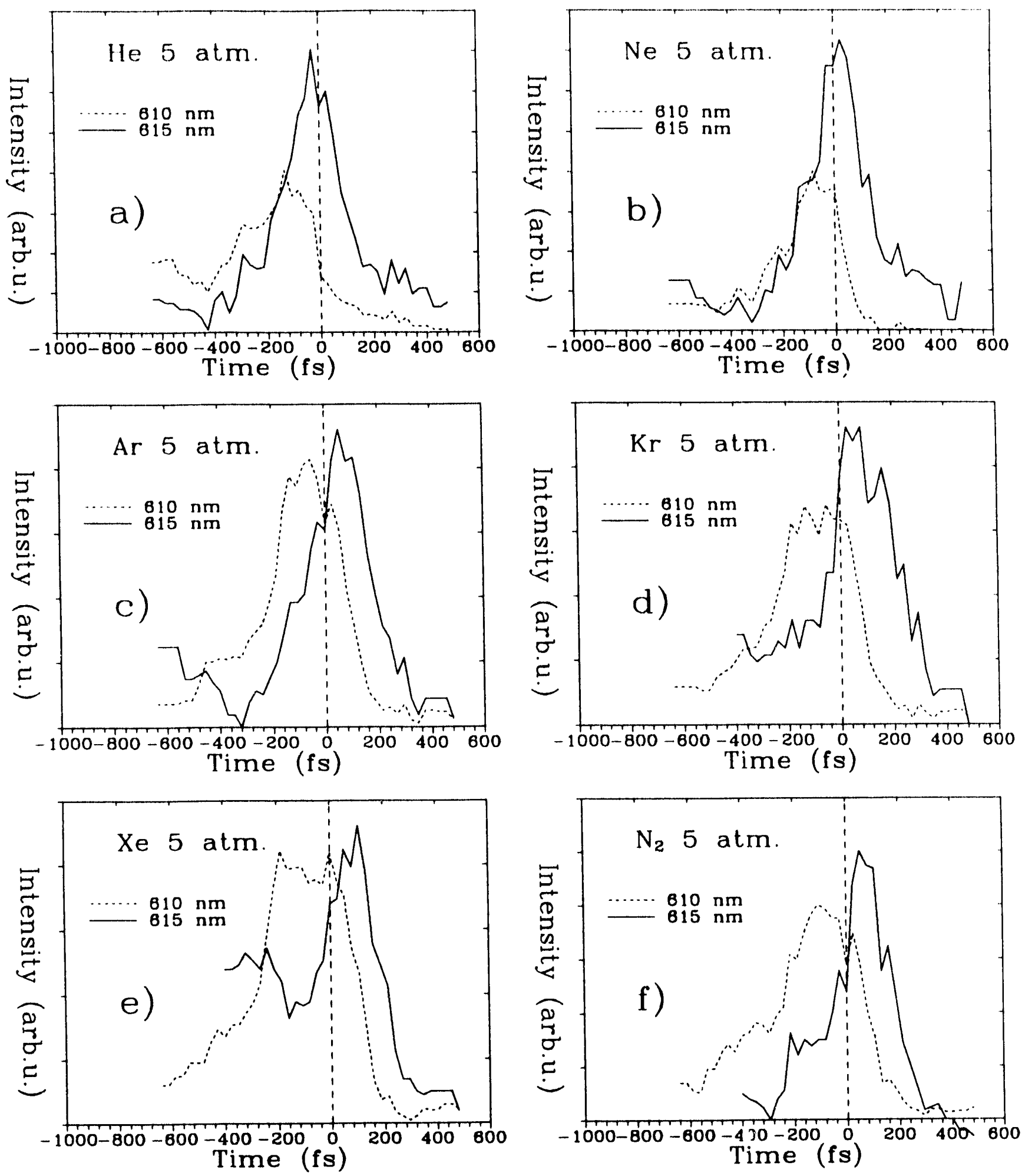
Femtosecond Growth Dynamics..., Wood, Siders, Downer
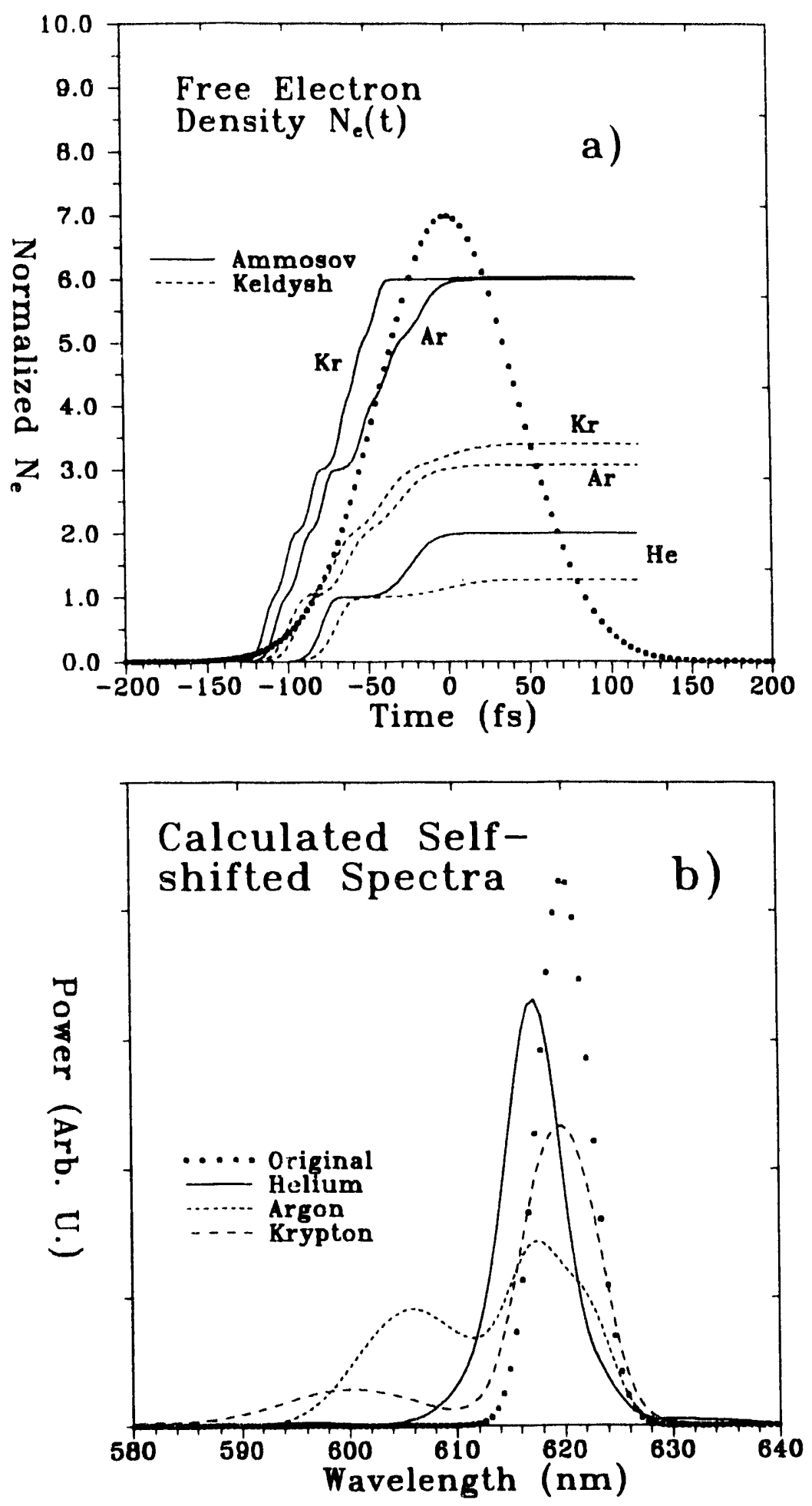
Femtosecond Growth Dynamics..., Wood, Siders, Downer
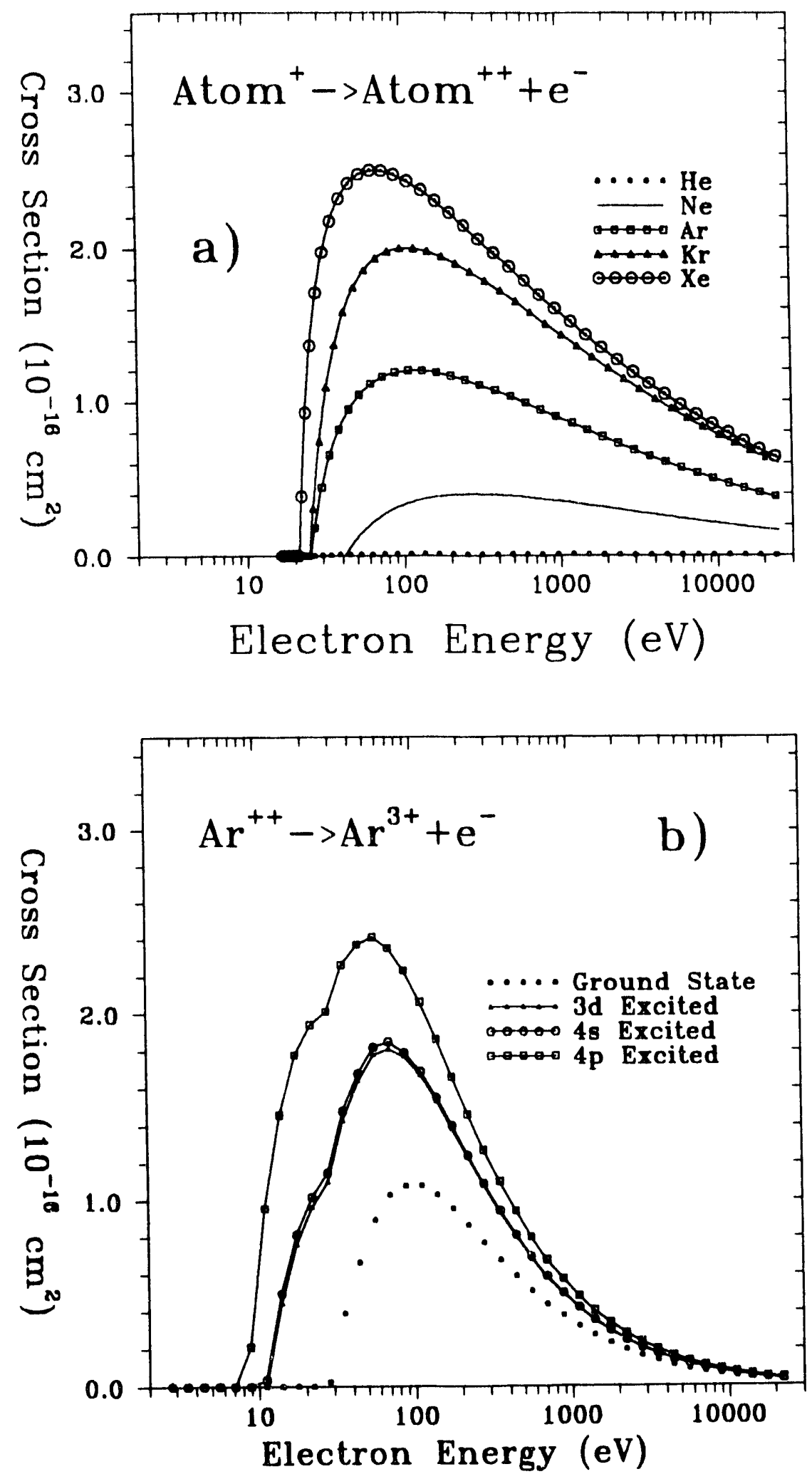
Femtosecond Growth Dynamics..., Wood, Siders, Downer
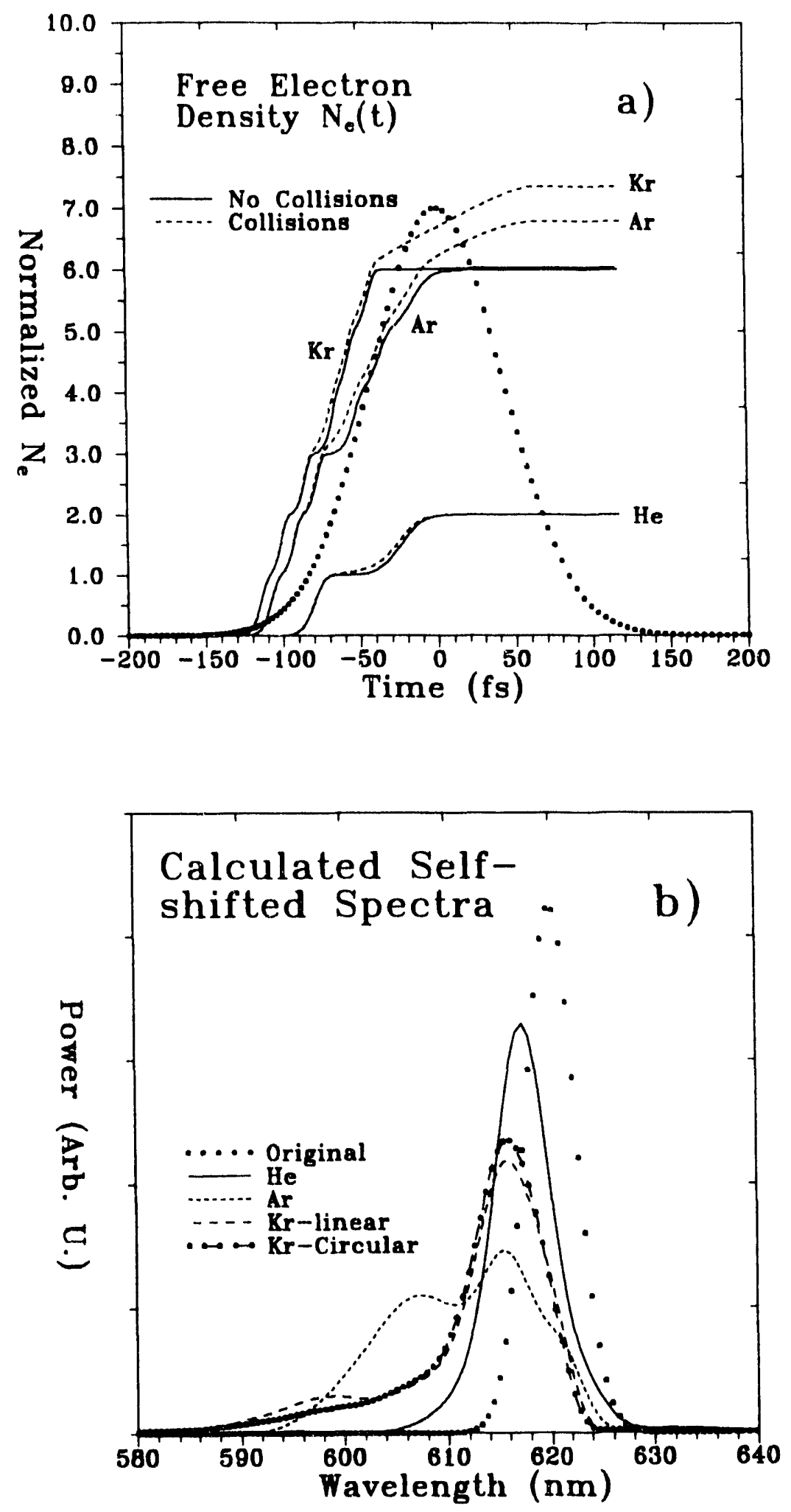
Femtosecond Growth Dynamics..., Wood, Siders, Downer
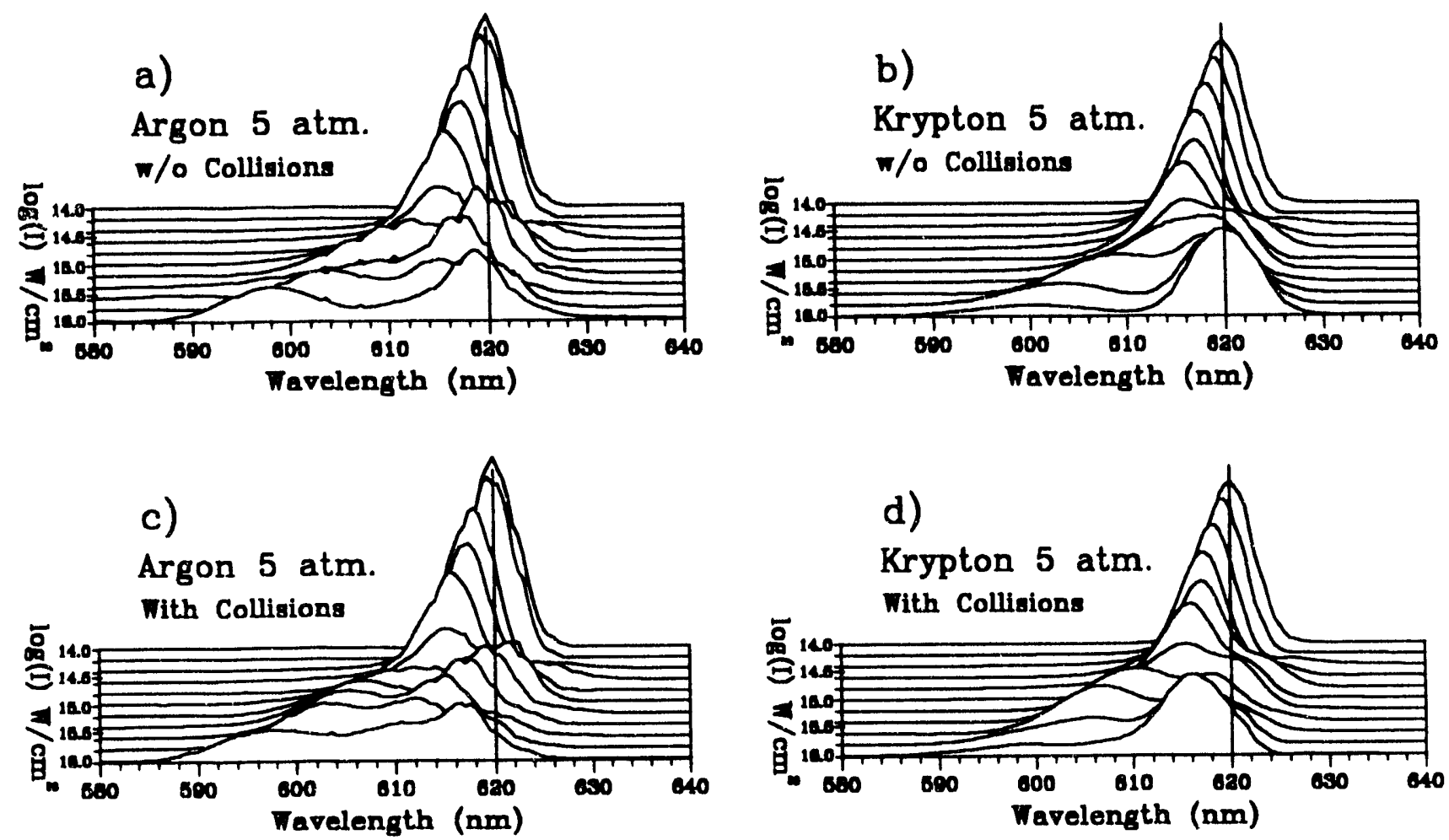
Femtosecond Growth Dynamics..., Wood, Siders, Downer
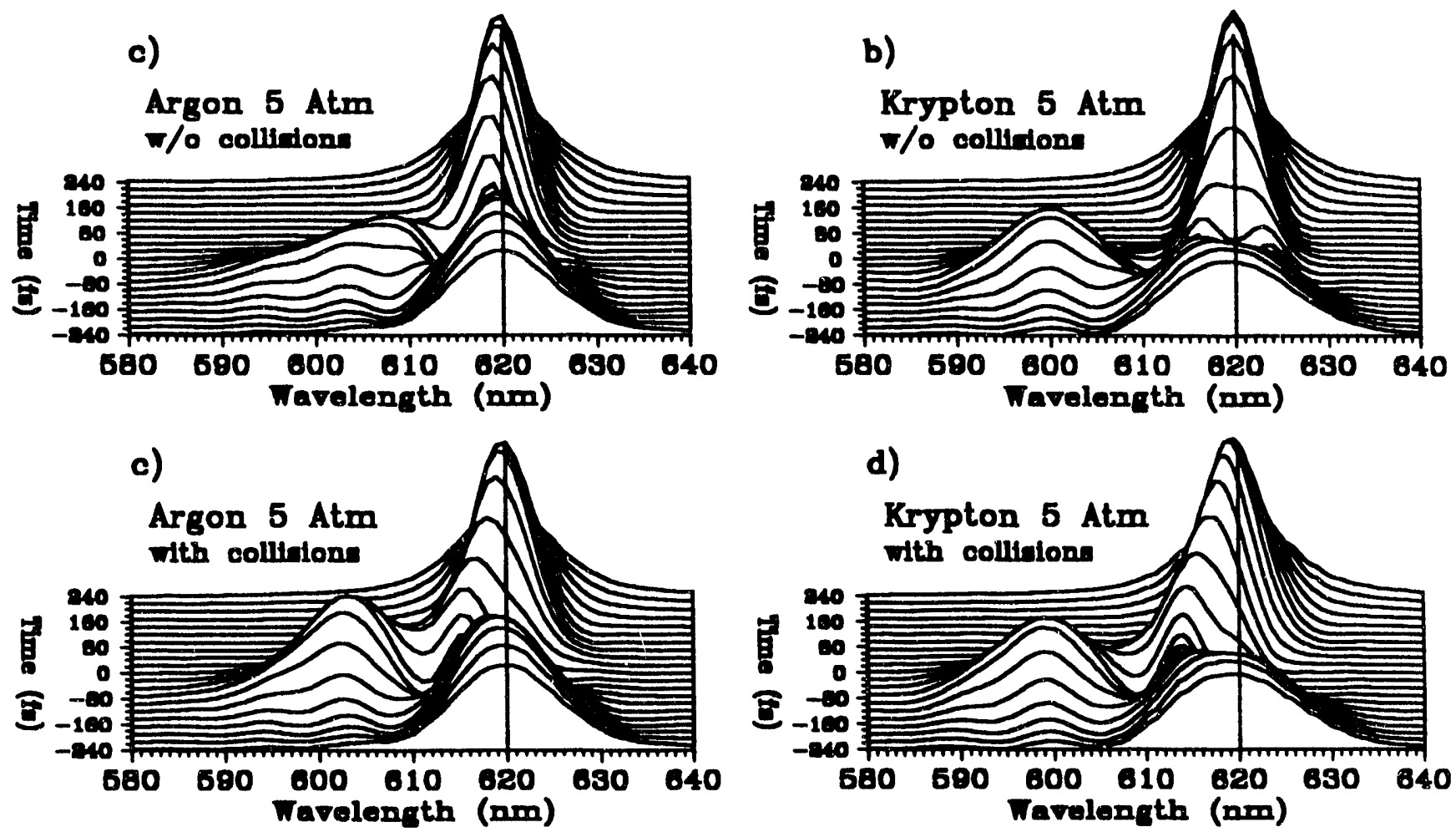
Femtosecond Growth Dynamics..., Wood, Siders, Downer
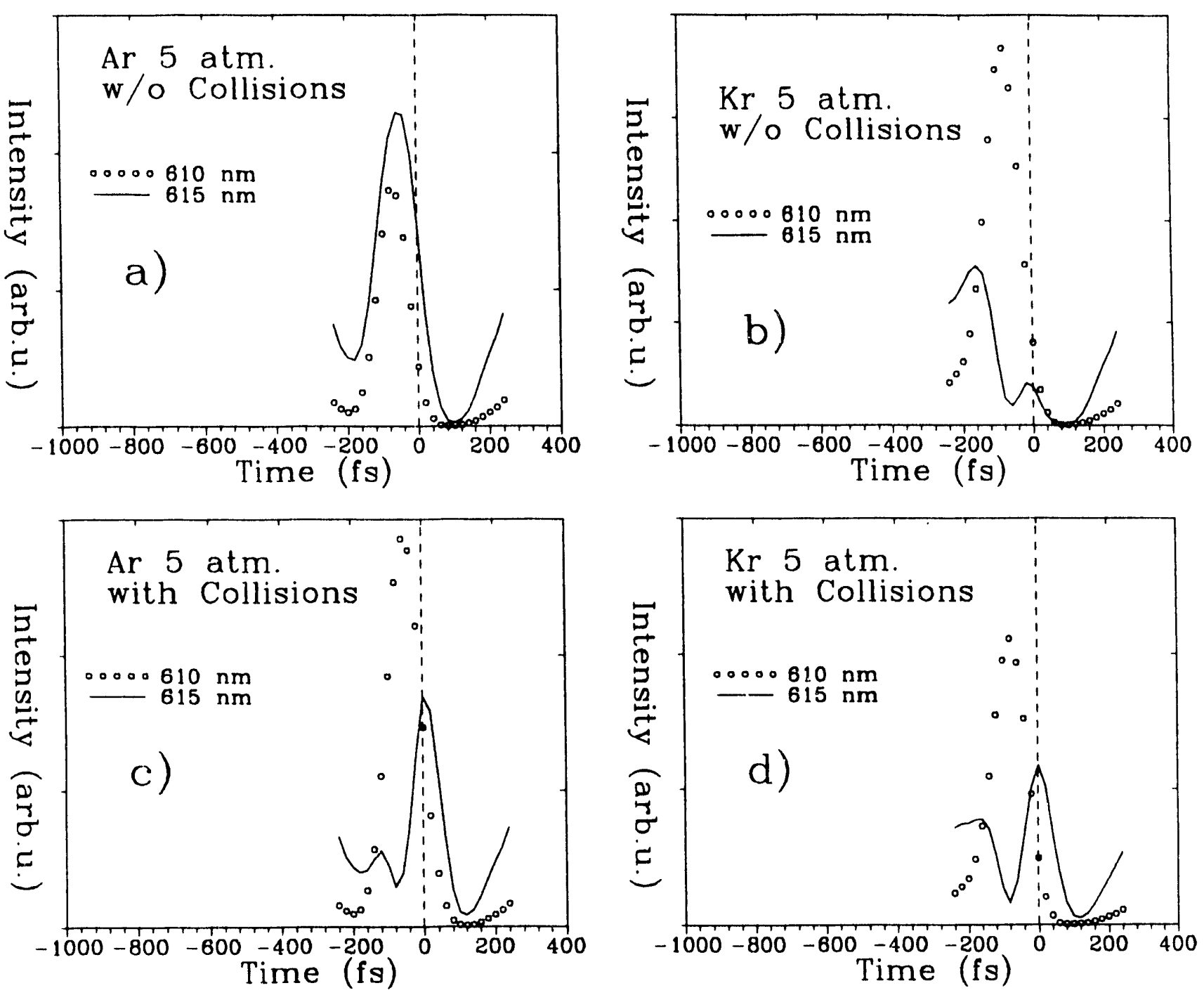

\section{DISCLAIMER}

This report was prepared as an account of work sponsored by an agency of the United States Government. Neither the United States Government nor any agency thereof, nor any of their employees, makes any warranty, express or implied, or assumes any legal liability or responsibility for the accuracy, completeness, or usefulness of any information, apparatus, product, or process disclosed, or represents that its use would not infringe privately owned rights. Reference herein to any specific commercial product, process, or service by trade name, trademark, manufacturer, or otherwise does not necessarily constitute or imply its endorsement, recommendation, or favoring by the United States Government or any agency thereof. The views and opinions of authors expressed herein do not necessarily state or reflect those of the United States Government or any agency thereof. 

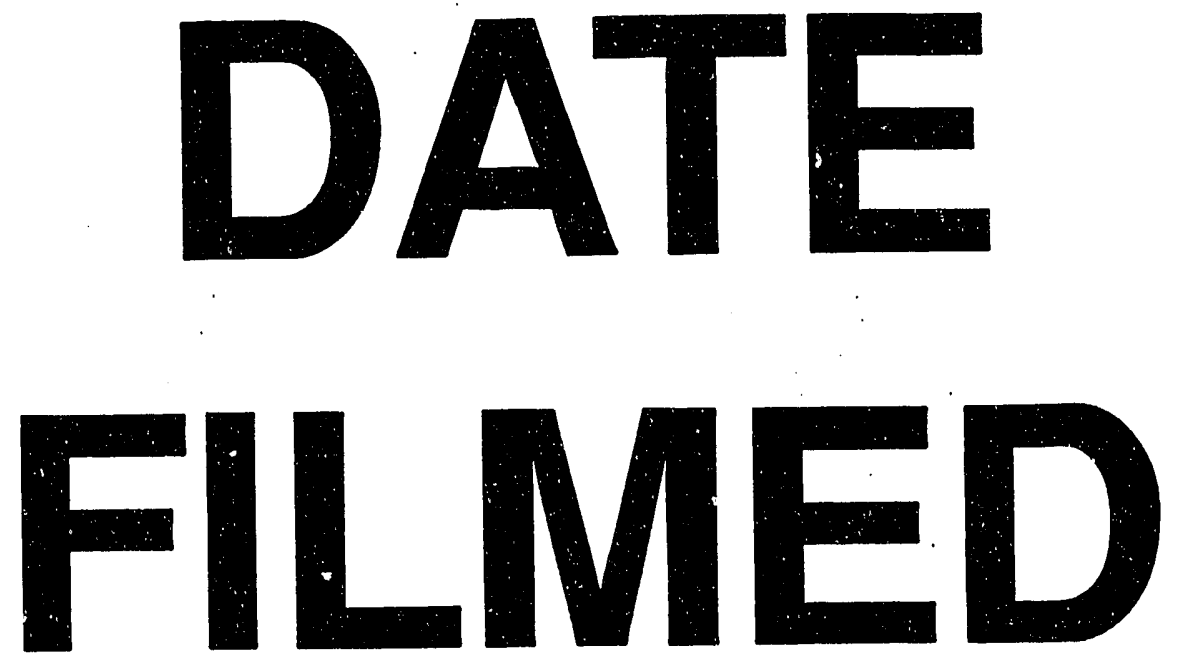

$8 / 17 / 93$
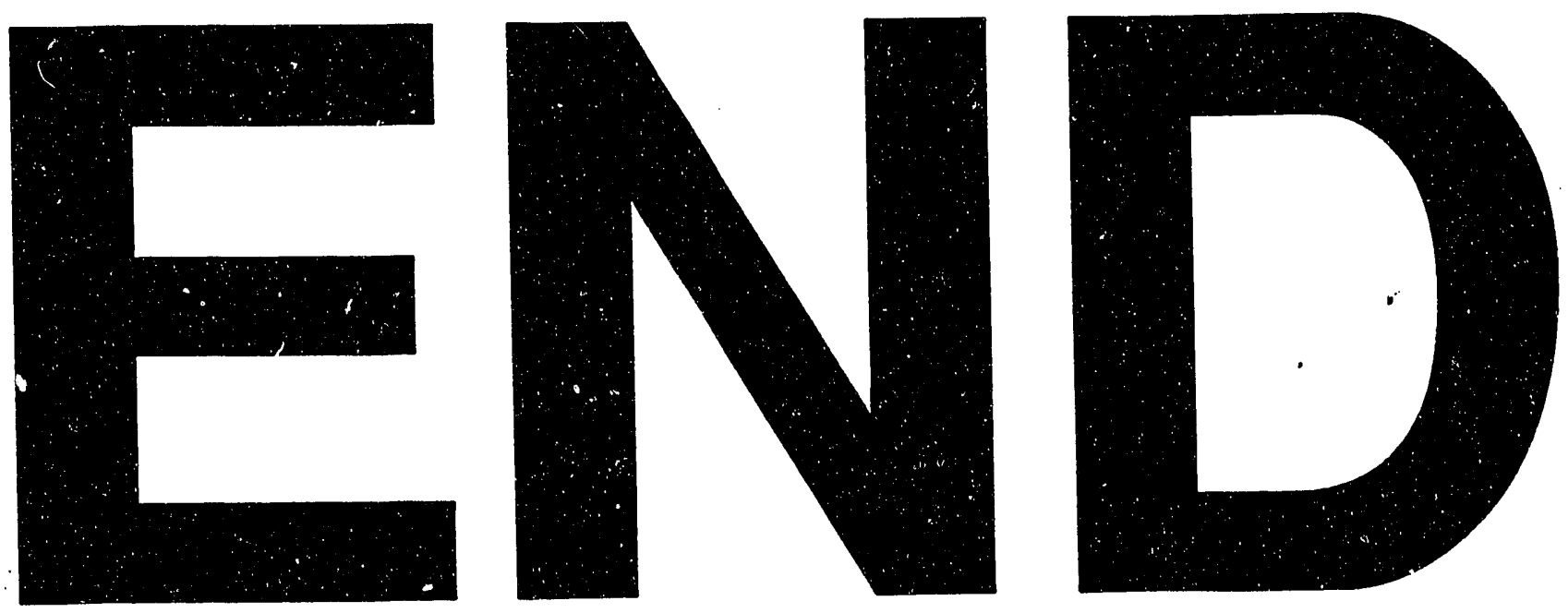
